ESAIM: M2AN

Vol. 42, $\mathrm{N}^{\mathrm{O}} 1,2008$, pp. 113-139

DOI: $10.1051 / \mathrm{m} 2 \mathrm{an}: 2007058$
ESAIM: Mathematical Modelling and Numerical Analysis

www.esaim-m2an.org

\title{
ANALYSIS OF A FORCE-BASED QUASICONTINUUM APPROXIMATION*
}

\author{
Matthew Dobson ${ }^{1}$ And Mitchell $\operatorname{Luskin}^{1}$
}

\begin{abstract}
We analyze a force-based quasicontinuum approximation to a one-dimensional system of atoms that interact by a classical atomistic potential. This force-based quasicontinuum approximation can be derived as the modification of an energy-based quasicontinuum approximation by the addition of nonconservative forces to correct nonphysical "ghost" forces that occur in the atomistic to continuum interface during constant strain. The algorithmic simplicity and consistency with the purely atomistic model at constant strain has made the force-based quasicontinuum approximation popular for largescale quasicontinuum computations. We prove that the force-based quasicontinuum equations have a unique solution when the magnitude of the external forces satisfy explicit bounds. For Lennard-Jones next-nearest-neighbor interactions, we show that unique solutions exist for external forces that extend the system nearly to its tensile limit. We give an analysis of the convergence of the ghost force iteration method to solve the equilibrium equations for the force-based quasicontinuum approximation. We show that the ghost force iteration is a contraction and give an analysis for its convergence rate.
\end{abstract}

Mathematics Subject Classification. 65Z05, 70C20.

Received December 15, 2006. Revised June 6, 2007.

\section{INTRODUCTION}

The local lattice structure for minimum energy configurations of atomistic systems subject to external forces is usually slowly varying except near defects such as dislocations [14]. Quasicontinuum methods efficiently approximate these multiscale features by maintaining atomistic degrees of freedom near defects and coarsegraining the atomistic degrees of freedom in regions where the local lattice structure is nearly uniform through the introduction of representative atoms $[10,14,24]$. The efficiency of quasicontinuum methods has allowed the simulation of more complex problems than can be computed using a completely atomistic model [22].

Many quasicontinuum methods have been proposed $[8,10,14,15,18,20,23,24]$, and each version gives a different quasicontinuum approximation of the atomistic system. A force-based quasicontinuum approximation has been proposed that modifies an energy-based quasicontinuum approximation by the addition of nonconservative forces to correct nonphysical "ghost" forces that occur in the atomistic to continuum interface $[10,14,20,22]$. The force-based quasicontinuum approximation has been popular for large-scale computations because it correctly computes forces under linear deformations and has a simple force computation: the force on each representative atom comes from either an atomistic algorithm or a continuum finite element algorithm [10,14,19,20,22].

Keywords and phrases. Quasicontinuum, ghost force, atomistic to continuum.

* This work was supported in part by NSF DMS-0304326 and by the University of Minnesota Supercomputing Institute. This work is also based on work supported by the Department of Energy under Award Number DE-FG02-05ER25706.

1 School of Mathematics, University of Minnesota, 206 Church Street SE, Minneapolis, MN 55455, USA. dobson@math.umn.edu; luskin@umn.edu

(C) EDP Sciences, SMAI 2008 
Adaptive mesh and error control have been successfully used with the force-based quasicontinuum approximation to efficiently choose representative atoms $[10,16,19,22]$. The number of representative atoms surrounding defects that need to be modeled atomistically (the core of the defect) can be determined by the error tolerance, and the mesh in the continuum region surrounding the core can be coarsened beyond the atomistic-continuum interface wherever the deformation gradient varies slowly. For simplicity, reported implementations have not coarsened within the cut-off radius of atomistic representative atoms, but it is possible to coarsen immediately beyond the atomistic-continuum interface by interpolating between continuum representative atoms.

In Section 2, we give a derivation following $[10,14,20,22]$ of several quasicontinuum approximations leading to the derivation of the force-based quasicontinuum approximation. In Section 3, we reformulate the equilibrium equations as a balance of forces conjugate to the distances between representative atoms, rather than as a balance of forces conjugate to the positions of the representative atoms. Our derivation and reformulation gives the mathematical structure that is used in our analysis.

In Section 4, we prove that the force-based quasicontinuum equations have a unique solution under suitable restrictions on the loads. This result is presented in Corollary 4.1. In the case of Lennard-Jones next-nearestneighbor interactions, we determine bounds for the magnitude of the loads for which unique solutions exist and find that the allowable loads extend quite close to the tensile limit.

In Section 5, we give an analysis of the convergence of the ghost force iteration method that has been most commonly used to solve the equilibrium equations for the force-based quasicontinuum approximation $[10,14,20,22]$. Corollary 4.1 shows that the ghost force iteration is a contraction and gives a bound for its convergence rate. We show that the convergence rate is quite high in the case of a Lennard-Jones model subject to moderate external forces.

Mathematical analyses of energy-based versions of the quasicontinuum approximation that do not include ghost force corrections have been given in $[2,5-7,11,12,17,18]$, and a simplified version of our analysis can be used to prove the existence of solutions to these energy-based quasicontinuum approximations. We show, though, that the ghost forces are nonconservative forces, so they cannot be derived from an energy. Thus, the force-based quasicontinuum approximation cannot be completely analyzed by energy methods.

We refer to [3] for a review of current progress on the mathematical analysis of atomistic to continuum models for solids and to [14] for an introduction and overview of the quasicontinuum approximation.

\section{QUASICONTINUUM APPROXIMATIONS}

In this section, we describe a sequence of one-dimensional coarse-grained approximations of a chain of atoms with nearest-neighbor and next-nearest-neighbor interactions given by a classical two-body potential, $\phi(r)$. We assume that the atomistic potential $\phi(r)$ is defined for all $r>0$.

We begin with the atomistic model, which has degrees of freedom for all atomic positions and computes a total internal energy directly from pairwise interactions. From there, we examine the constrained atomistic approximation and the local quasicontinuum approximation which both decrease the degrees of freedom by interpolating atomic positions between representative atoms. We then introduce an energy-based and a force-based quasicontinuum approximation which span atomistic and continuum scales by combining atomistic regions where the atoms directly interact according to the atomistic model and continuum regions where the atoms interact according to the local quasicontinuum approximation. We observe that the energy-based quasicontinuum approximation gives nonphysical ghost forces near the atomistic to continuum interface that are corrected by the force-based quasicontinuum approximation.

\subsection{The atomistic model}

We denote the positions of the atoms by $y_{i}$ for $i=-M, \ldots, M+1$, where $y_{i}<y_{i+1}$ (see Fig. 1). The total energy for the atomistic system with nearest-neighbor and next-nearest-neighbor interactions given by 


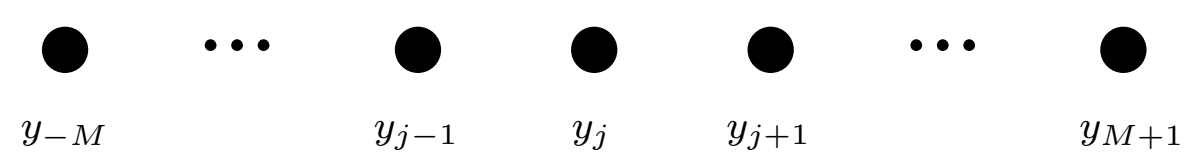

Figure 1. Atomistic chain with atoms labeled by their position.

the classical two-body potential $\phi(r)$ is

$$
\mathcal{E}^{a}(\mathbf{y})=\sum_{i=-M}^{M}\left[\phi\left(y_{i+1}-y_{i}\right)+\phi\left(y_{i+2}-y_{i}\right)\right],
$$

where $\mathbf{y}=\left(y_{-M}, \ldots, y_{M+1}\right) \in \mathbb{R}^{2 M+2}$ and where the boundary terms $\phi\left(y_{i}-y_{j}\right)$ above and in the following should be understood to be zero for $i \notin\{-M, \ldots, M+1\}$ or $j \notin\{-M, \ldots, M+1\}$. We can also express the total energy in terms of energies associated with each atom as

$$
\mathcal{E}^{a}(\mathbf{y})=\sum_{i=-M}^{M+1} \mathcal{E}_{i}^{a}(\mathbf{y})
$$

with

$$
\mathcal{E}_{i}^{a}(\mathbf{y})=\frac{1}{2}\left[\phi\left(y_{i+1}-y_{i}\right)+\phi\left(y_{i+2}-y_{i}\right)+\phi\left(y_{i}-y_{i-1}\right)+\phi\left(y_{i}-y_{i-2}\right)\right] .
$$

We then have that the force on the atom at position $y_{i}$ is given by

$$
\begin{aligned}
F_{i}^{a}(\mathbf{y}) & =-\frac{\partial}{\partial y_{i}}\left[\phi\left(y_{i+1}-y_{i}\right)+\phi\left(y_{i+2}-y_{i}\right)+\phi\left(y_{i}-y_{i-1}\right)+\phi\left(y_{i}-y_{i-2}\right)\right] \\
& =\left[\eta\left(r_{i}\right)+\eta\left(r_{i}+r_{i+1}\right)\right]-\left[\eta\left(r_{i-1}\right)+\eta\left(r_{i-1}+r_{i-2}\right)\right]
\end{aligned}
$$

where $\eta(r)=\phi^{\prime}(r)$ and $r_{i}=y_{i+1}-y_{i}$ is the lattice spacing at $y_{i}$. The terms $\eta\left(r_{i}\right)$ and $\eta\left(r_{i}+r_{j}\right)$ above and in the following should be understood to be zero for $i \notin\{-M, \ldots, M\}$ or $j \notin\{-M, \ldots, M\}$.

We now assume that the atoms are also subject to an external force, $\tilde{f}_{i}\left(y_{i}\right)$, that is obtained from an external potential energy of the form

so

$$
\mathcal{P}^{a}(\mathbf{y})=\sum_{i=-M}^{M+1} \mathcal{P}_{i}^{a}\left(y_{i}\right)
$$

$$
\tilde{f}_{i}\left(y_{i}\right)=-\frac{\partial \mathcal{P}^{a}(\mathbf{y})}{\partial y_{i}}=-\frac{\partial \mathcal{P}_{i}^{a}\left(y_{i}\right)}{\partial y_{i}}
$$

For example, such external forces may model the interaction of the one-dimensional chain with atoms in layers above and below the chain, as in the Frenkel Kontorova model [13].

We then have the equilibrium equations

$$
F_{i}^{a}(\mathbf{y})+\tilde{f}_{i}\left(y_{i}\right)=0, \quad i=-M, \ldots, M+1
$$

\subsection{The constrained atomistic quasicontinuum approximation}

One can reduce the degrees of freedom in the atomistic model by linearly interpolating the positions of the atoms between a set of representative atoms (see [18] for an analysis in this case). We introduce representative atoms with positions $z_{j}$ such that

$$
z_{j}=y_{\ell_{j}} \quad \text { for } j=-N, \ldots, N+1 \text {, }
$$




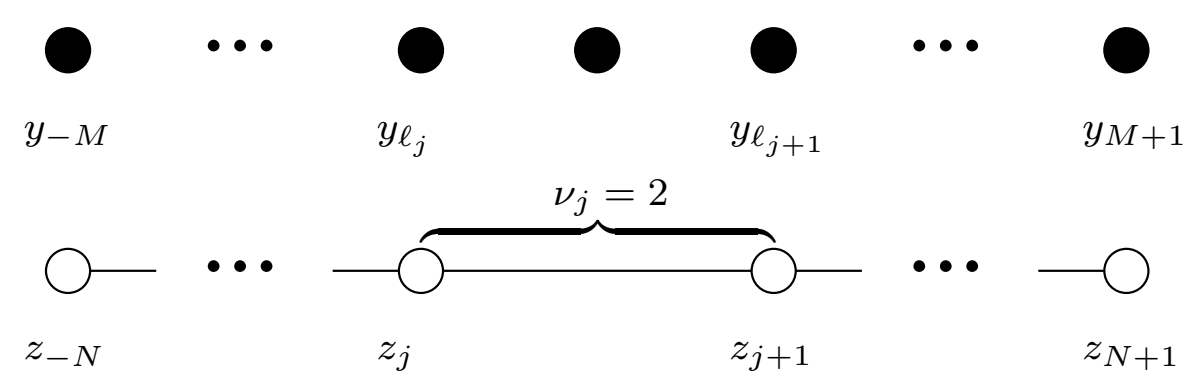

Figure 2. A coarsening of the atomistic chain.

where $\ell_{-N}=-M, \ell_{N+1}=M+1$, and $\ell_{j}<\ell_{j+1}$. We then let $\nu_{j}=\ell_{j+1}-\ell_{j}$ denote the number of atoms from $z_{j}$ to $z_{j+1}$ (where the end atoms at $z_{j}$ and $z_{j+1}$ are counted as half, see Fig. 2). We now have that

$$
r_{j}=\frac{z_{j+1}-z_{j}}{\nu_{j}}
$$

is the distance separating $\left(\nu_{j}+1\right)$ equally spaced atoms from $z_{j}$ to $z_{j+1}$, and we have the conservation of mass equation

$$
\sum_{j=-N}^{N} \nu_{j}=\sum_{j=-N}^{N}\left(\ell_{j+1}-\ell_{j}\right)=\ell_{N+1}-\ell_{-N}=2 M+1 .
$$

In the constrained atomistic model, the positions of atoms between $z_{j}$ and $z_{j+1}$ are linearly interpolated as

$$
y_{\ell_{j}+i}=y_{\ell_{j+1}-\left(\nu_{j}-i\right)}=\frac{\nu_{j}-i}{\nu_{j}} z_{j}+\frac{i}{\nu_{j}} z_{j+1} \quad \text { for } 0 \leq i \leq \nu_{j}
$$

and we define the total internal energy in terms of $\mathbf{z}=\left(z_{-N}, \ldots, z_{N+1}\right) \in \mathbb{R}^{2 N+2}$ to again be the interaction energy of all atoms in the chain, computed according to (2.1), giving

$$
\mathcal{E}^{c}(\mathbf{z})=\sum_{j=-M}^{M+1} \mathcal{E}_{j}^{a}(\mathbf{y}(\mathbf{z})) .
$$

So far, we have reduced the degrees of freedom necessary for denoting the atomistic positions, but the total energy is still computed as a sum of energy contributions from all of the atomistic degrees of freedom. However, all nearest-neighbor and next-nearest-neighbor contributions for atoms interpolated between a pair of representative atoms are identical due to the uniform spacing, so if $y_{i}$ does not denote a representative atom we have that

$$
\phi\left(y_{i+1}-y_{i-1}\right)=\phi\left(2\left(y_{i+1}-y_{i}\right)\right)=\phi\left(2\left(y_{i}-y_{i-1}\right)\right)=\phi\left(2 r_{j}\right) \quad \text { for } \ell_{j}<i<\ell_{j+1}
$$

These interactions account for all of the energy contributions except for next-nearest-neighbor interactions that straddle a representative atom. We can treat these interactions by observing that

$$
\begin{gathered}
\phi\left(y_{i+1}-y_{i-1}\right)-\frac{1}{2} \phi\left(2\left(y_{i}-y_{i-1}\right)\right)-\frac{1}{2} \phi\left(2\left(y_{i+1}-y_{i}\right)\right)= \\
\begin{cases}\phi\left(r_{j-1}+r_{j}\right)-\frac{1}{2} \phi\left(2 r_{j-1}\right)-\frac{1}{2} \phi\left(2 r_{j}\right), & \text { if } i=\ell_{j} \text { for some } j=-N, \ldots, N+1, \\
0, & \text { if } i \neq \ell_{j} \text { for all } j=-N, \ldots, N+1 .\end{cases}
\end{gathered}
$$


We can therefore partition the total energy into the energy of the region between $z_{j}$ and $z_{j+1}$ for each $j=-N, \ldots, N$, plus interfacial energy terms that account for interactions that straddle a representative atom. We have from (2.8) that

$$
\begin{aligned}
\mathcal{E}^{c}(\mathbf{z})= & \sum_{i=-M}^{M} \phi\left(y_{i+1}-y_{i}\right)+\sum_{i=-M+1}^{M} \phi\left(y_{i+1}-y_{i-1}\right) \\
= & \sum_{i=-M}^{M}\left[\phi\left(y_{i+1}-y_{i}\right)+\phi\left(2\left(y_{i+1}-y_{i}\right)\right)\right] \\
& +\sum_{i=-M+1}^{M}\left[\phi\left(y_{i+1}-y_{i-1}\right)-\frac{1}{2} \phi\left(2\left(y_{i}-y_{i-1}\right)\right)-\frac{1}{2} \phi\left(2\left(y_{i+1}-y_{i}\right)\right)\right] \\
& -\frac{1}{2} \phi\left(2\left(y_{-M+1}-y_{-M}\right)\right)-\frac{1}{2} \phi\left(2\left(y_{M+1}-y_{M}\right)\right) \\
= & \sum_{j=-N}^{N} \nu_{j} \hat{\phi}\left(r_{j}\right)+\sum_{j=-N}^{N+1} \mathcal{S}_{j}\left(r_{j-1}, r_{j}\right),
\end{aligned}
$$

where

and where

$$
\hat{\phi}(r)=\phi(r)+\phi(2 r) \text { for } r>0
$$

$$
\begin{aligned}
\mathcal{S}_{-N}\left(r_{-N}\right) & =-\frac{1}{2} \phi\left(2 r_{-N}\right), \\
\mathcal{S}_{j}\left(r_{j-1}, r_{j}\right) & =-\frac{1}{2} \phi\left(2 r_{j-1}\right)+\phi\left(r_{j-1}+r_{j}\right)-\frac{1}{2} \phi\left(2 r_{j}\right), \quad j=-N+1, \ldots, N, \\
\mathcal{S}_{N+1}\left(r_{N}\right) & =-\frac{1}{2} \phi\left(2 r_{N}\right) .
\end{aligned}
$$

We note that $\hat{\phi}(r)=\phi(r)+\phi(2 r)$ is the energy per atom for an infinite atomistic chain with the uniform lattice spacing $y_{i+1}-y_{i}=r$ for all $-\infty<i<\infty$, and that $\mathcal{S}_{j}\left(r_{j-1}, r_{j}\right)$ can be considered to be a surface energy at $z_{-N}$ and $z_{N+1}$ and to be an interfacial energy at $z_{j}$ for $j=-N+1, \ldots, N$. We observe that $\mathcal{S}_{j}\left(r_{j-1}, r_{j}\right)$ is a second divided difference for $\phi(r)$ about $r=r_{j-1}+r_{j}$ with increment $r_{j}-r_{j-1}$, so

$$
\mathcal{S}_{j}\left(r_{j-1}, r_{j}\right)=-\frac{1}{2} \phi^{\prime \prime}\left(r_{j-1}+r_{j}\right)\left(r_{j}-r_{j-1}\right)^{2}+O\left(\left|r_{j}-r_{j-1}\right|^{4}\right), \quad j=-N+1, \ldots, N .
$$

We will see that if $\phi(r)$ satisfies the assumptions given in Section 4, then the convexity condition

$$
\mathcal{S}_{j}\left(r_{j-1}, r_{j}\right)>0, \quad j=-N+1, \ldots, N
$$

holds for the range of $\mathbf{r}=\left(r_{-N}, \ldots, r_{N}\right) \in \mathbb{R}^{2 N+1}$ defined by (4.15) in Theorem 4.1 and Corollary 4.1 where solutions of the force-based quasicontinuum equilibrium equations are shown to exist and where the iterates of the ghost force iteration reside.

\subsection{The local quasicontinuum approximation}

If we neglect the surface and interfacial energy terms, $\mathcal{S}_{j}$, in $(2.9)$, then we obtain the local quasicontinuum approximation [14]

$$
\mathcal{E}^{L}(\mathbf{z})=\sum_{j=-N}^{N+1} \mathcal{E}_{j}^{L}(\mathbf{z}),
$$


where

$$
\mathcal{E}_{j}^{L}(\mathbf{z})=\frac{1}{2}\left[\hat{\phi}\left(r_{j}\right) \nu_{j}+\hat{\phi}\left(r_{j-1}\right) \nu_{j-1}\right] .
$$

To treat the boundary terms consistently, we set $\nu_{-N-1}=\nu_{N+1}=1$ and $\hat{\phi}\left(r_{-N-1}\right)=\hat{\phi}\left(r_{N+1}\right)=0$.

We remark that the representative atoms need not be placed at atomistic sites as described by (2.5). The local quasicontinuum approximation only requires that $z_{j}<z_{j+1}$ for $j=-N, \ldots, N$ and that the $\nu_{j}$ are positive and satisfy the conservation of mass condition (2.6). The approximation can be generalized to higher space dimensions by using the Cauchy-Born rule [14]. The local quasicontinuum approximation is computationally simpler than the constrained atomistic quasicontinuum approximation, especially in higher dimensions where the computation of the interfacial energy becomes expensive. In the following, we will sometimes refer to the local quasicontinuum approximation as the continuum approximation.

The force on a representative atom at $z_{j}$ for $j=-N+1, \ldots, N$ is given for the local quasicontinuum approximation by

$$
\begin{aligned}
F_{j}^{L}(\mathbf{z})= & -\frac{\partial}{\partial z_{j}}\left[\hat{\phi}\left(\frac{z_{j+1}-z_{j}}{\nu_{j}}\right) \nu_{j}+\hat{\phi}\left(\frac{z_{j}-z_{j-1}}{\nu_{j-1}}\right) \nu_{j-1}\right] \\
= & -\frac{\partial}{\partial z_{j}}\left[\phi\left(\frac{z_{j+1}-z_{j}}{\nu_{j}}\right) \nu_{j}+\phi\left(\frac{2\left(z_{j+1}-z_{j}\right)}{\nu_{j}}\right) \nu_{j}\right. \\
& \left.+\phi\left(\frac{z_{j}-z_{j-1}}{\nu_{j-1}}\right) \nu_{j-1}+\phi\left(\frac{2\left(z_{j}-z_{j-1}\right)}{\nu_{j-1}}\right) \nu_{j-1}\right] \\
= & {\left[\eta\left(r_{j}\right)+2 \eta\left(2 r_{j}\right)\right]-\left[\eta\left(r_{j-1}\right)+2 \eta\left(2 r_{j-1}\right)\right], }
\end{aligned}
$$

where again

$$
r_{j}=\frac{\left(z_{j+1}-z_{j}\right)}{\nu_{j}}
$$

is the lattice constant for the atoms from $z_{j}$ to $z_{j+1}$. In the above, we see the "local" nature of the approximation, as the force on a degree of freedom is determined only by the positions of adjacent degrees of freedom and no long-range interactions occur.

We can similarly compute the force on the boundary atoms, noting the one-sided nature of $\mathcal{E}_{j}^{L}(\mathbf{z})$ for the boundary atoms, by

$$
\begin{aligned}
F_{-N}^{L}(\mathbf{z})= & -\frac{\partial}{\partial z_{-N}}\left[\hat{\phi}\left(\frac{z_{-N+1}-z_{-N}}{\nu_{-N}}\right) \nu_{-N}\right] \\
= & -\frac{\partial}{\partial z_{-N}}\left[\phi\left(\frac{z_{-N+1}-z_{-N}}{\nu_{-N}}\right)+\phi\left(\frac{2\left(z_{-N+1}-z_{-N}\right)}{\nu_{-N}}\right)\right] \nu_{-N} \\
= & \left.\eta \eta\left(r_{-N}\right)+2 \eta\left(2 r_{-N}\right)\right], \\
F_{N+1}^{L}(\mathbf{z})= & -\frac{\partial}{\partial z_{N+1}}\left[\hat{\phi}\left(\frac{z_{N+1}-z_{N}}{\nu_{N}}\right) \nu_{N}\right] \\
= & -\frac{\partial}{\partial z_{N+1}}\left[\phi\left(\frac{z_{N+1}-z_{N}}{\nu_{N}}\right)+\phi\left(\frac{2\left(z_{N+1}-z_{N}\right)}{\nu_{N}}\right)\right] \nu_{N} \\
= & -\left[\eta\left(r_{N}\right)+2 \eta\left(2 r_{N}\right)\right] .
\end{aligned}
$$

We note that the local quasicontinuum energy, $\mathcal{E}^{L}(\mathbf{z})$, and the forces, $F_{j}^{L}(\mathbf{z})$, depend only on $\mathbf{r}=\left(r_{-N}, \ldots, r_{N}\right)$, and we will denote the dependence by $\mathcal{E}^{L}(\mathbf{r})$ and $F_{j}^{L}(\mathbf{r})$ without introducing distinct functions.

We can also derive a local quasicontinuum approximation for the external potential, $\mathcal{P}^{L}(\mathbf{z})$, by setting

$$
\mathcal{P}^{L}(\mathbf{z})=\mathcal{P}^{a}(\mathbf{y}(\mathbf{z}))
$$




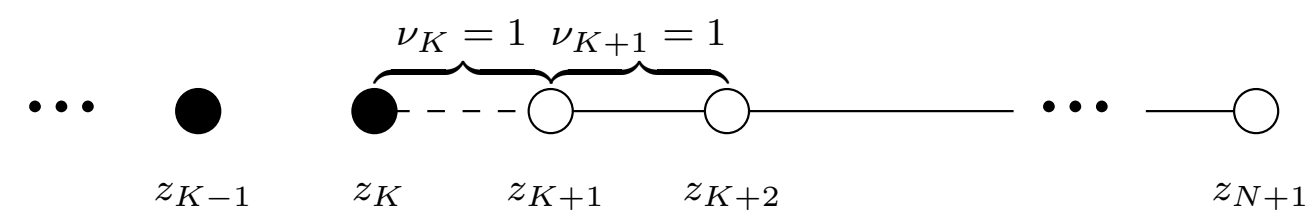

FIGURE 3. One end of the quasicontinuum chain, highlighting the interface. Filled circles are atomistic representative atoms, whereas the unfilled circles are continuum representative atoms.

By (2.4) and (2.7), the external force on the representative atom at position $z_{j}$ is

$$
\begin{aligned}
f_{j}(\mathbf{z})=-\frac{\partial \mathcal{P}^{L}(\mathbf{z})}{\partial z_{j}} & =-\sum_{i=0}^{\nu_{j-1}}\left(\frac{\nu_{j-1}-i}{\nu_{j-1}}\right) \frac{\partial \mathcal{P}^{a}(\mathbf{y}(\mathbf{z}))}{\partial y_{\ell_{j}-i}}-\sum_{i=1}^{\nu_{j}}\left(\frac{\nu_{j}-i}{\nu_{j}}\right) \frac{\partial \mathcal{P}^{a}(\mathbf{y}(\mathbf{z}))}{\partial y_{\ell_{j}+i}} \\
& =\sum_{i=0}^{\nu_{j-1}}\left(\frac{\nu_{j-1}-i}{\nu_{j-1}}\right) \tilde{f}_{\ell_{j}-i}\left(y_{\ell_{j}-i}(\mathbf{z})\right)+\sum_{i=1}^{\nu_{j}}\left(\frac{\nu_{j}-i}{\nu_{j}}\right) \tilde{f}_{\ell_{j}+i}\left(y_{\ell_{j}+i}(\mathbf{z})\right) .
\end{aligned}
$$

It follows from the linear interpolation (2.7) that

$$
f_{j}(\mathbf{z})=f_{j}\left(z_{j-1}, z_{j}, z_{j+1}\right)
$$

We shall assume in our analysis that the external forces, $\tilde{f}_{i}$, are independent of $\mathbf{y}$. In this case, the local quasicontinuum forces, $f_{j}$, are independent of $\mathbf{z}$ and

$$
f_{j}=\sum_{i=0}^{\nu_{j-1}}\left(\frac{\nu_{j-1}-i}{\nu_{j-1}}\right) \tilde{f}_{\ell_{j}-i}+\sum_{i=1}^{\nu_{j}}\left(\frac{\nu_{j}-i}{\nu_{j}}\right) \tilde{f}_{\ell_{j}+i}, \quad j=-N, \ldots, N+1
$$

We consider any term $\tilde{f}_{j}$ to be zero if $j \notin\{-M, \ldots, M+1\}$ and any term $\nu_{j}$ to be one if $j \notin\{-N, \ldots, N\}$.

\subsection{The energy-based quasicontinuum approximation}

To describe the energy-based quasicontinuum approximation [14], we again introduce representative atoms with positions $z_{j}$ for $j=-N, \ldots, N+1$, where $z_{j}<z_{j+1}$. Each representative atom is considered to be an "atomistic" or "continuum" degree of freedom and will contribute either $\mathcal{E}_{j}^{a}(\mathbf{z})$ or $\mathcal{E}_{j}^{L}(\mathbf{z})$ to the total internal energy according to the atomistic model (2.2) or the local quasicontinuum approximation (2.11), respectively.

In applications, atomistic degrees of freedom are used in regions of interest where highly non-uniform behavior is expected. Continuum regions surround this, gradually coarsening by increasing $\nu_{j}$ in regions with slowly varying strain. For simplicity of exposition, we will consider an approximation with a single atomistic region, symmetrically surrounded by continuum regions large enough so that no atomistic degrees of freedom interact with the surface atoms through nearest-neighbor or next-nearest-neighbor interactions.

We denote the representative atom positions by $z_{j}$ and define the range $j=-K+1, \ldots, K$ to be atomistic sites and the ranges $j=-N, \ldots,-K$ and $K+1, \ldots, N+1$ to be continuum sites (see Fig. 3). Therefore, the total quasicontinuum energy, $\mathcal{E}^{Q C}(\mathbf{r})$, for the chain is given by

$$
\mathcal{E}^{Q C}(\mathbf{r})=\sum_{j=-N}^{-K} \mathcal{E}_{j}^{L}(\mathbf{r})+\sum_{j=-K+1}^{K} \mathcal{E}_{j}^{a}(\mathbf{r})+\sum_{j=K+1}^{N+1} \mathcal{E}_{j}^{L}(\mathbf{r})
$$

where $\mathcal{E}_{j}^{a}(\mathbf{r})$ is defined in $(2.2)$ and $\mathcal{E}_{j}^{L}(\mathbf{r})$ is defined in (2.11). We assume that $\nu_{j}=1$ for $j=-K-1, \ldots, K+1$. This guarantees that $\nu_{j}=1$ within the next-nearest-neighbor cutoff radius of any atomistic site and enables a seamless transition to the continuum approximation. 
The force, $F_{j}^{Q C E}(\mathbf{r})$, at $z_{j}$ is then given by

$$
\begin{aligned}
F_{j}^{Q C E}(\mathbf{r})= & -\frac{\partial \mathcal{E}^{Q C}}{\partial z_{j}}(\mathbf{r})=-\sum_{\ell=-N}^{N} \frac{\partial \mathcal{E}^{Q C}}{\partial r_{\ell}}(\mathbf{r}) \frac{\partial r_{\ell}}{\partial z_{j}} \\
& = \begin{cases}F_{j}^{L}(\mathbf{r}), & -N \leq j \leq-K-2, \\
F_{-K-1}^{L}(\mathbf{r})+\frac{1}{2} \eta\left(r_{-K-1}+r_{-K}\right), & j=-K-1, \\
F_{-K}^{L}(\mathbf{r})-\eta\left(2 r_{-K}\right)+\frac{1}{2} \eta\left(r_{-K}+r_{-K+1}\right), & j=-K, \\
F_{-K+1}^{a}(\mathbf{r})-\eta\left(2 r_{-K}\right)+\frac{1}{2} \eta\left(r_{-K-1}+r_{-K}\right), & j=-K+1, \\
F_{-K+2}^{a}(\mathbf{r})+\frac{1}{2} \eta\left(r_{-K}+r_{-K+1}\right), & j=-K+2, \\
F_{j}^{a}(\mathbf{r}), & -K+3 \leq j \leq K-2, \\
F_{K-1}^{a}(\mathbf{r})-\frac{1}{2} \eta\left(r_{K-1}+r_{K}\right), & j=K-1, \\
F_{K}^{a}(\mathbf{r})+\eta\left(2 r_{K}\right)-\frac{1}{2} \eta\left(r_{K}+r_{K+1}\right), & j=K, \\
F_{K+1}^{L}(\mathbf{r})+\eta\left(2 r_{K}\right)-\frac{1}{2} \eta\left(r_{K-1}+r_{K}\right), & j=K+1, \\
F_{K+2}^{L}(\mathbf{r})-\frac{1}{2} \eta\left(r_{K}+r_{K+1}\right), & j=K+2, \\
F_{j}^{L}(\mathbf{r}), & K+3 \leq j \leq N+1 .\end{cases}
\end{aligned}
$$

In the above expression, we notice that in the large ranges interior to the atomistic and continuum regions the forces are exactly those from the individual models, namely, either $F_{j}^{a}(\mathbf{r})$ or $F_{j}^{L}(\mathbf{r})$. Near the atomistic to continuum interface, there are additions to these force terms which contain non-physical "ghost" forces.

To see this, we consider the forces on the representative atoms when the lattice spacings are uniform, that is, when $r_{j}=a$ for $j=-N, \ldots, N$, or equivalently when $\mathbf{r}=\mathbf{a}=(a, \ldots, a) \in \mathbb{R}^{2 N+1}$. We observe that the forces computed according to the atomistic model (2.3) or the continuum model (2.12) are zero except at the ends of the chain, that is, $F_{j}^{L}(\mathbf{a})=0$ for $j=-N+1, \ldots, N$ and $F_{j}^{a}(\mathbf{a})=0$ for $j=-N+2, \ldots, N-1$. We then have from (2.18) that

$$
F_{j}^{Q C E}(\mathbf{a})= \begin{cases}{[\eta(a)+2 \eta(2 a)],} & j=-N, \\ 0, & -N+1 \leq j \leq-K-2, \\ \frac{1}{2} \eta(2 a), & j=-K-1, \\ -\frac{1}{2} \eta(2 a), & j=-K, \\ -\frac{1}{2} \eta(2 a), & j=-K+1, \\ \frac{1}{2} \eta(2 a), & j=-K+2, \\ 0, & -K+3 \leq j \leq K-2, \\ -\frac{1}{2} \eta(2 a), & j=K-1, \\ \frac{1}{2} \eta(2 a), & j=K, \\ \frac{1}{2} \eta(2 a), & j=K+1, \\ -\frac{1}{2} \eta(2 a), & j=K+2, \\ 0, & K+3 \leq j \leq N, \\ -[\eta(a)+2 \eta(2 a)], & j=N+1 .\end{cases}
$$

Figure 4 shows the ghost forces at one of the interfaces. Figure 5 shows the origin of out of balance forces for a single representative atom. We note that while the first neighbor terms are balanced, the second neighbor terms are not balanced due to the fact that the continuum site does not contribute any second-neighbor interactions.

The force-based quasicontinuum approximation described in the next subsection corrects these ghost forces. 


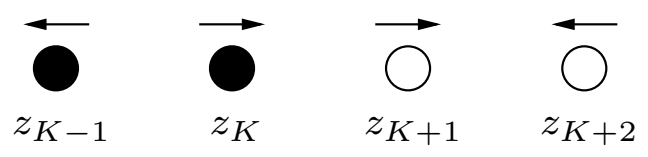

FIGURE 4. Direction of nonzero forces for a uniform configuration.

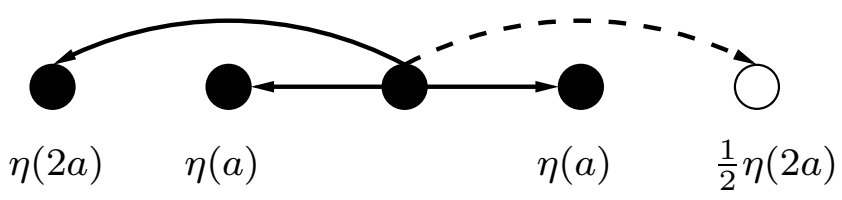

FiguRE 5. Imbalance of forces on an atomistic representative atom near the interface.

\subsection{The force-based quasicontinuum approximation}

The force-based quasicontinuum approximation [14] corrects the nonphysical forces described in the previous subsection. The forces on the representative atoms in the interior of the atomistic and continuum regions are defined by (2.3) and (2.12), but the forces on the representative atoms near the atomistic-continuum transition given by the energy-based quasicontinuum method must be modified to remove the non-physical terms.

In the force-based quasicontinuum approximation, we again partition into atomistic and continuum representative atoms, where the force on a representative atom is the force that would result on it if the approximation was entirely of its respective type (atomistic or continuum). With this convention, a continuum representative atom only interacts with adjacent degrees of freedom regardless of how close any atomistic sites may be. We will see that the tradeoff for this simple philosophy is that the forces are not conservative, that is, they cannot be derived from an energy.

We now model the forces on the representative atoms for $j=-K+1, \ldots, K$ where $K<N-1$ by the atomistic model (2.3) and the forces on the representative atoms for $j=-N, \ldots,-K$ and $j=K+1, \ldots, N+1$ by the local quasicontinuum approximation (2.12)-(2.14). The forces on the representative atoms are then given by

$$
\begin{aligned}
F_{j}^{Q C F}(\mathbf{r})= & \begin{cases}F_{j}^{L}(\mathbf{r}), \quad-N \leq j \leq-K, \\
F_{j}^{a}(\mathbf{r}), \quad-K+1 \leq j \leq K, \\
F_{j}^{L}(\mathbf{r}), \quad K+1 \leq j \leq N+1,\end{cases} \\
= & \begin{array}{ll}
{\left[\eta\left(r_{-N}\right)+2 \eta\left(2 r_{-N}\right)\right],} & j=-N, \\
{\left[\eta\left(r_{j}\right)+2 \eta\left(2 r_{j}\right)\right]-\left[\eta\left(r_{j-1}\right)+2 \eta\left(2 r_{j-1}\right)\right],} & -N+1 \leq j \leq-K, \\
{\left[\eta\left(r_{j}\right)+\eta\left(r_{j}+r_{j+1}\right)\right]-\left[\eta\left(r_{j-1}\right)+\eta\left(r_{j-1}+r_{j-2}\right)\right],} & -K+1 \leq j \leq K, \\
{\left[\eta\left(r_{j}\right)+2 \eta\left(2 r_{j}\right)\right]-\left[\eta\left(r_{j-1}\right)+2 \eta\left(2 r_{j-1}\right)\right],} & K+1 \leq j \leq N, \\
-\left[\eta\left(r_{N}\right)+2 \eta\left(2 r_{N}\right)\right], & j=N+1 .
\end{array}
\end{aligned}
$$

The force-based quasicontinuum formulation has the desired property that if we take a uniform configuration, $\mathbf{a}=(a, \ldots, a) \in \mathbb{R}^{2 N+1}$, we have that

$$
F_{j}^{Q C F}(\mathbf{a})=0, \quad j=-N+1, \ldots, N
$$

and on the boundary we get values of equal magnitude and opposite signs. Thus, the equilibrium equations have a uniform solution, a, whenever equal and opposite external forces are applied at the boundary, that is, a chain in uniform tension or compression. 
However, the solution to the equilibrium equations for the force-based quasicontinuum method,

$$
F_{j}^{Q C F}(\mathbf{r})+f_{j}=0, \quad j=-N, \ldots, N+1,
$$

cannot be obtained from the minimization of an energy since $F_{j}^{Q C F}(\mathbf{r})$ is a nonconservative force. To see this, we observe that the forces given by the force-based quasicontinuum approximation, $F_{j}^{Q C F}(\mathbf{r})$, are not the differential of an energy, $\mathcal{E}^{Q C F}(\mathbf{r})$, since $F_{j}^{Q C F}(\mathbf{r})$ is not a closed form [9]. We can see this by noting that

$$
\begin{aligned}
& \frac{\partial F_{K}^{Q C F}}{\partial z_{K+1}}(\mathbf{r})=\eta^{\prime}\left(r_{K}\right), \\
& \frac{\partial F_{K+1}^{Q C F}}{\partial z_{K}}(\mathbf{r})=\eta^{\prime}\left(r_{K}\right)+4 \eta^{\prime}\left(2 r_{K}\right),
\end{aligned}
$$

implies that

$$
\frac{\partial F_{K}^{Q C F}}{\partial z_{K+1}}(\mathbf{r}) \neq \frac{\partial F_{K+1}^{Q C F}}{\partial z_{K}}(\mathbf{r})
$$

\subsection{The ghost force iteration}

In the quasicontinuum method of [14], the forces, $F^{Q C F}(\mathbf{r})$, are split into the force from the energy-based quasicontinuum approximation, $F^{Q C E}(\mathbf{r})$, and ghost force corrections defined by

$$
F_{j}^{G}(\mathbf{r})=F_{j}^{Q C F}(\mathbf{r})-F_{j}^{Q C E}(\mathbf{r})= \begin{cases}0, & -N \leq j \leq-K-2, \\ -\frac{1}{2} \eta\left(r_{-K-1}+r_{-K}\right), & j=-K-1, \\ +\eta\left(2 r_{-K}\right)-\frac{1}{2} \eta\left(r_{-K}+r_{-K+1}\right), & j=-K, \\ +\eta\left(2 r_{-K}\right)-\frac{1}{2} \eta\left(r_{-K-1}+r_{-K}\right), & j=-K+1, \\ -\frac{1}{2} \eta\left(r_{-K}+r_{-K+1}\right), & j=-K+2, \\ 0, & -K+3 \leq j \leq K-2, \\ +\frac{1}{2} \eta\left(r_{K-1}+r_{K}\right), & j=K-1, \\ -\eta\left(2 r_{K}\right)+\frac{1}{2} \eta\left(r_{K}+r_{K+1}\right), & j=K, \\ -\eta\left(2 r_{K}\right)+\frac{1}{2} \eta\left(r_{K-1}+r_{K}\right), & j=K+1, \\ +\frac{1}{2} \eta\left(r_{K}+r_{K+1}\right), & j=K+2, \\ 0, & K+3 \leq j \leq N+1 .\end{cases}
$$

The forces $F_{j}^{G}(\mathbf{r})$ act as a model correction near the atomistic-continuum interfaces to enforce the convention that each representative atom has forces acting on it as though it were surrounded by representative atoms of the same type.

In [14], the solution to the equilibrium equations $(\mathrm{F})$ is obtained by solving the iteration

$$
F_{j}^{Q C E}\left(\mathbf{r}^{n+1}\right)+F_{j}^{G}\left(\mathbf{r}^{n}\right)+f_{j}=0, \quad j=-N, \ldots, N+1,
$$

by using a conjugate gradient method to compute $\mathbf{r}^{n+1}$ from $\mathbf{r}^{n}$. If the sequence of solutions $\left\{\mathbf{r}^{n}\right\}$ converges, then the iterative limit $\mathbf{r}$ satisfies the equilibrium equations $(\mathrm{F})$.

We have split the internal forces of the force-based quasicontinuum approximation, $F_{j}^{Q C F}(\mathbf{r})$, into a conservative force, $F_{j}^{Q C E}(\mathbf{r})$, and correction, $F_{j}^{G}(\mathbf{r})$, to define the iterative method above (E). The conservative force, $F_{j}^{Q C E}(\mathbf{r})$, was defined by the quasicontinuum energy, $\mathcal{E}^{Q C}(\mathbf{r})$, given by $(2.17)$, and the correction, $F_{j}^{G}(\mathbf{r})$, 
was defined simply by $F_{j}^{G}(\mathbf{r})=F_{j}^{Q C F}(\mathbf{r})-F_{j}^{Q C E}(\mathbf{r})$. However, we are free to try to improve the rate of convergence of $\mathbf{r}^{n} \rightarrow \mathbf{r}$ by constructing an energy $\mathcal{E}^{Q C E 2}(\mathbf{r})$ different from $\mathcal{E}^{Q C E}(\mathbf{r})$ and then solving the iteration

$$
F_{j}^{Q C E 2}\left(\mathbf{r}^{n+1}\right)+F_{j}^{G 2}\left(\mathbf{r}^{n}\right)+f_{j}=0, \quad j=-N, \ldots, N+1,
$$

where $F_{j}^{Q C E 2}(\mathbf{r})$ is now the force that is derived from $\mathcal{E}^{Q C E 2}(\mathbf{r})$ and $F_{j}^{G 2}(\mathbf{r})=F_{j}^{Q C F}(\mathbf{r})-F_{j}^{Q C E 2}(\mathbf{r})$. In the following we restrict our attention to $\mathcal{E}^{Q C E}$ as presented in this section, leaving other choices to be addressed in future work.

\section{Conjugate forces}

In this section, we simplify the analysis by reformulating the force-based quasicontinuum equilibrium equations $(\mathrm{F})$ in terms of forces conjugate to the distance between representative atoms,

$$
R_{j}=z_{j+1}-z_{j}=\nu_{j} r_{j}, \quad j=-N, \ldots, N
$$

rather than conjugate to the positions of the representative atoms, $z_{j}$. We will use the notation $\mathbf{R}=$ $\left(R_{-N}, \ldots, R_{N}\right) \in \mathbb{R}^{2 N+1}$. Through this technique, we will be able to derive equations that are decoupled inside the continuum regions and are the sum of tridiagonal terms and nonlocal interfacial terms in the atomistic region. The main results, Theorems 4.1 and 5.1, will be in terms of the conjugate forces.

\subsection{The internal conjugate force}

We define the internal conjugate force for the energy-based quasicontinuum approximation by

$$
\psi_{j}^{E}(\mathbf{r})=\frac{\partial \mathcal{E}^{Q C}}{\partial R_{j}}(\mathbf{r}(\mathbf{R}))=\frac{1}{\nu_{j}} \frac{\partial \mathcal{E}^{Q C}}{\partial r_{j}}(\mathbf{r}), \quad j=-N, \ldots, N
$$

We note that we have found it convenient to define $\psi_{j}^{E}(\mathbf{r})$ as the negative of the usual convention for a conjugate force. We next derive the following relation between $F_{j}^{Q C E}(\mathbf{r})$ and the internal conjugate force $\psi_{j}^{E}(\mathbf{r})$ :

$$
\begin{aligned}
F_{j}^{Q C E}(\mathbf{r}) & =-\frac{\partial \mathcal{E}^{Q C}}{\partial z_{j}}(\mathbf{r})=-\frac{\partial \mathcal{E}^{Q C}}{\partial R_{j}}(\mathbf{r}(\mathbf{R})) \frac{\partial R_{j}}{\partial z_{j}}-\frac{\partial \mathcal{E}^{Q C}}{\partial R_{j-1}}(\mathbf{r}(\mathbf{R})) \frac{\partial R_{j-1}}{\partial z_{j}} \\
& =\psi_{j}^{E}(\mathbf{r})-\psi_{j-1}^{E}(\mathbf{r}), \quad j=-N, \ldots, N+1,
\end{aligned}
$$

where we set

$$
\psi_{-N-1}^{E}(\mathbf{r})=\psi_{N+1}^{E}(\mathbf{r})=0 .
$$

We can sum the forces from the left of the chain and use the preceding equation (3.2) to obtain that

$$
\psi_{j}^{E}(\mathbf{r})=\sum_{i=-N}^{j} F_{i}^{Q C E}(\mathbf{r}), \quad j=-N, \ldots, N+1 .
$$


We can derive from either (3.1) or (3.3) that

$$
\psi_{j}^{E}(\mathbf{r})= \begin{cases}0, & j=-N-1 \\ \eta\left(r_{j}\right)+2 \eta\left(2 r_{j}\right), & -N \leq j \leq-K-2, \\ \eta\left(r_{j}\right)+2 \eta\left(2 r_{j}\right)+\frac{1}{2} \eta\left(r_{j}+r_{j+1}\right), & j=-K-1, \\ \eta\left(r_{j}\right)+\frac{1}{2} \eta\left(r_{j}+r_{j-1}\right)+\frac{1}{2} \eta\left(r_{j}+r_{j+1}\right)+\eta\left(2 r_{j}\right), & j=-K, \\ \eta\left(r_{j}\right)+\frac{1}{2} \eta\left(r_{j}+r_{j-1}\right)+\eta\left(r_{j}+r_{j+1}\right), & j=-K+1, \\ \eta\left(r_{j}\right)+\eta\left(r_{j}+r_{j-1}\right)+\eta\left(r_{j}+r_{j+1}\right), & -K+2 \leq j \leq K-2, \\ \eta\left(r_{j}\right)+\eta\left(r_{j}+r_{j-1}\right)+\frac{1}{2} \eta\left(r_{j}+r_{j+1}\right), & j=K-1, \\ \eta\left(r_{j}\right)+\frac{1}{2} \eta\left(r_{j}+r_{j-1}\right)+\frac{1}{2} \eta\left(r_{j}+r_{j+1}\right)+\eta\left(2 r_{j}\right), & j=K, \\ \eta\left(r_{j}\right)+2 \eta\left(2 r_{j}\right)+\frac{1}{2} \eta\left(r_{j}+r_{j-1}\right), & j=K+1, \\ \eta\left(r_{j}\right)+2 \eta\left(2 r_{j}\right), & K+2 \leq j \leq N, \\ 0, & j=N+1 .\end{cases}
$$

We cannot properly derive an internal conjugate force for the force-based quasicontinuum approximation since it is not a conservative force. However, we will find it convenient to define one by following (3.3), setting

$$
\psi_{-N-1}^{F}(\mathbf{r})=0
$$

and

$$
\psi_{j}^{F}(\mathbf{r})=\sum_{i=-N}^{j} F_{i}^{Q C F}(\mathbf{r}), \quad j=-N, \ldots, N+1
$$

We can then obtain that

$$
F_{j}^{Q C F}(\mathbf{r})=\psi_{j}^{F}(\mathbf{r})-\psi_{j-1}^{F}(\mathbf{r}), \quad j=-N, \ldots, N+1
$$

We have the following closed form expressions for the internal conjugate force $\psi_{j}^{F}(\mathbf{r})$ :

$$
\psi_{j}^{F}(\mathbf{r})= \begin{cases}0, & j=-N-1, \\ \eta\left(r_{j}\right)+2 \eta\left(2 r_{j}\right), & -N \leq j \leq-K, \\ \eta\left(r_{j}\right)+\eta\left(r_{j}+r_{j-1}\right)+\eta\left(r_{j}+r_{j+1}\right) & \\ \quad+\left[2 \eta\left(2 r_{-K}\right)-\eta\left(r_{-K}+r_{-K-1}\right)-\eta\left(r_{-K}+r_{-K+1}\right)\right], & -K+1 \leq j \leq K, \\ \eta\left(r_{j}\right)+2 \eta\left(2 r_{j}\right) & \\ \quad+\left[2 \eta\left(2 r_{-K}\right)-\eta\left(r_{-K}+r_{-K-1}\right)-\eta\left(r_{-K}+r_{-K+1}\right)\right] & \\ -\left[2 \eta\left(2 r_{K}\right)-\eta\left(r_{K}+r_{K-1}\right)-\eta\left(r_{K}+r_{K+1}\right)\right], & K+1 \leq j \leq N, \\ {\left[2 \eta\left(2 r_{-K}\right)-\eta\left(r_{-K}+r_{-K-1}\right)-\eta\left(r_{-K}+r_{-K+1}\right)\right]} & \\ \quad-\left[2 \eta\left(2 r_{K}\right)-\eta\left(r_{K}+r_{K-1}\right)-\eta\left(r_{K}+r_{K+1}\right)\right], & j=N+1 .\end{cases}
$$

The internal conjugate force, $\psi_{j}^{F}(\mathbf{r})$, takes a simpler form when there is no resultant quasicontinuum force, that is, when $\mathbf{r}$ satisfies

which is equivalent to

$$
\psi_{N+1}^{F}(\mathbf{r})=\sum_{j=-N}^{N+1} F_{j}^{Q C F}(\mathbf{r})=0
$$

$$
2 \eta\left(2 r_{-K}\right)-\eta\left(r_{-K}+r_{-K-1}\right)-\eta\left(r_{-K}+r_{-K+1}\right)=2 \eta\left(2 r_{K}\right)-\eta\left(r_{K}+r_{K-1}\right)-\eta\left(r_{K}+r_{K+1}\right) .
$$


We note that (3.9) is satisfied when $\mathbf{r}$ is symmetric, that is, when $r_{-j}=r_{j}$ for $j=1, \ldots, N$. Let us now define $\hat{\psi}_{j}^{F}(\mathbf{r})$ on all of $\mathbb{R}^{2 N+1}$ as a symmetric extension of $\psi_{j}^{F}(\mathbf{r})$, with equality whenever $\mathbf{r}$ satisfies (3.9). This leads to $\hat{\psi}_{j}^{F}(\mathbf{r})$ having a more symmetric form,

$$
\hat{\psi}_{j}^{F}(\mathbf{r})= \begin{cases}0, & j=-N-1, \\ \eta\left(r_{j}\right)+2 \eta\left(2 r_{j}\right), & -N \leq j \leq-K, \\ \eta\left(r_{j}\right)+\eta\left(r_{j}+r_{j-1}\right)+\eta\left(r_{j}+r_{j+1}\right) & \\ \quad+\left[2 \eta\left(2 r_{K}\right)-\eta\left(r_{K}+r_{K-1}\right)-\eta\left(r_{K}+r_{K+1}\right)\right], & -K+1 \leq j \leq K-1, \\ \eta\left(r_{j}\right)+2 \eta\left(2 r_{j}\right), & K \leq j \leq N, \\ 0, & j=N+1 .\end{cases}
$$

The intervals of definition changed slightly from (3.7), which is one of the simplifications afforded by (3.9). We use $\hat{\psi}_{j}^{F}(\mathbf{r})$ in our subsequent analysis, and in Section 3.3 we discuss the relation between using $\psi_{j}^{F}(\mathbf{r})$ and $\hat{\psi}_{j}^{F}(\mathbf{r})$ to solve the equilibrium equations $(\mathrm{F})$.

We identify in (3.10) a continuum internal conjugate force for $j=-N, \ldots,-K$ and $j=K, \ldots, N$ given by

$$
\eta\left(r_{j}\right)+2 \eta\left(2 r_{j}\right)
$$

and an atomistic internal conjugate force for $j=-K+1, \ldots, K-1$ given by

$$
\eta\left(r_{j}\right)+\eta\left(r_{j-1}+r_{j}\right)+\eta\left(r_{j}+r_{j+1}\right) .
$$

We identify the remaining terms,

$$
2 \eta\left(2 r_{K}\right)-\eta\left(r_{K}+r_{K-1}\right)-\eta\left(r_{K}+r_{K+1}\right),
$$

for $j=-K+1, \ldots, K-1$ as the nonlocal part of the internal conjugate force.

For consistency, we wish to define $\hat{\psi}_{j}^{E}(\mathbf{r})$ as we did for $\hat{\psi}_{j}^{F}(\mathbf{r})$. Since $\psi_{j}^{E}(\mathbf{r})$ is derived from the energy $\mathcal{E}^{Q C E}(\mathbf{r})$, it has no resultant force. Thus, we define $\hat{\psi}_{j}^{E}(\mathbf{r})=\psi_{j}^{E}(\mathbf{r})$.

We can also derive a corresponding internal conjugate ghost force, $\psi_{j}^{G}(\mathbf{r})$, by summing $F_{j}^{G}(\mathbf{r})$ as in (3.5). From the definition of $F_{j}^{G}(\mathbf{r})(2.19)$, we have that

$$
\psi_{j}^{F}(\mathbf{r})=\psi_{j}^{E}(\mathbf{r})+\psi_{j}^{G}(\mathbf{r}), \quad j=-N-1, \ldots, N+1 .
$$

We can define $\hat{\psi}_{j}^{G}(\mathbf{r})$ by

and we can then check that

$$
\hat{\psi}_{j}^{F}(\mathbf{r})=\hat{\psi}_{j}^{E}(\mathbf{r})+\hat{\psi}_{j}^{G}(\mathbf{r}), \quad j=-N-1, \ldots, N+1,
$$

$$
\hat{\psi}_{j}^{G}(\mathbf{r})= \begin{cases}0, & -N-1 \leq j \leq-K-2, \\ -\frac{1}{2} \eta\left(r_{-K}+r_{-K-1}\right), & j=-K-1, \\ \eta\left(2 r_{-K}\right)-\frac{1}{2} \eta\left(r_{-K}+r_{-K+1}\right)-\frac{1}{2} \eta\left(r_{-K}+r_{-K-1}\right), & j=-K, \\ 2 \eta\left(2 r_{-K}\right)-\frac{1}{2} \eta\left(r_{-K}+r_{-K+1}\right)-\eta\left(r_{-K}+r_{-K-1}\right), & j=-K+1, \\ 2 \eta\left(2 r_{K}\right)-\eta\left(r_{K}+r_{K-1}\right)-\eta\left(r_{K}+r_{K+1}\right), & -K+2 \leq j \leq K-2, \\ 2 \eta\left(2 r_{K}\right)-\frac{1}{2} \eta\left(r_{K}+r_{K-1}\right)-\eta\left(r_{K}+r_{K+1}\right), & j=K-1, \\ \eta\left(2 r_{K}\right)-\frac{1}{2} \eta\left(r_{K}+r_{K-1}\right)-\frac{1}{2} \eta\left(r_{K}+r_{K+1}\right), & j=K, \\ -\frac{1}{2} \eta\left(r_{K}+r_{K+1}\right), & j=K+1, \\ 0, & K+2 \leq j \leq N+1 .\end{cases}
$$


If $\mathbf{r}$ satisfies the condition (3.9), then we have that $\hat{\psi}_{j}^{G}(\mathbf{r})=\psi_{j}^{G}(\mathbf{r})$. We note that the nonlocal part of the internal conjugate force is found in $\psi_{j}^{G}(\mathbf{r})$ and $\hat{\psi}_{j}^{G}(\mathbf{r})$.

We finally observe that if $\mathbf{r}$ is symmetric, then $\hat{\psi}_{j}^{F}(\mathbf{r})=\psi_{j}^{F}(\mathbf{r}), \hat{\psi}_{j}^{E}(\mathbf{r})=\psi_{j}^{E}(\mathbf{r})$, and $\hat{\psi}_{j}^{G}(\mathbf{r})=\psi_{j}^{G}(\mathbf{r})$ are symmetric.

\subsection{The external conjugate force}

We recall that we are assuming in our analysis that the external forces, $\tilde{f}_{i}$, are independent of $\mathbf{y}$, and that consequently the local quasicontinuum forces, $f_{j}$, given in (2.16) are independent of $\mathbf{z}$. The external potential, $\mathcal{P}^{L}(\mathbf{z})$, given in (2.15) thus has the form

$$
\mathcal{P}^{L}(\mathbf{z})=-\sum_{j=-N}^{N+1} f_{j} z_{j}
$$

We now assume that there is also no resultant force from the external forces, so that

$$
\sum_{j=-N}^{N+1} f_{j}=0
$$

It then follows that the external potential, $\mathcal{P}^{L}(\mathbf{z})$, is also a function of $\mathbf{r}$, and we can define the external conjugate force by

$$
\Phi_{j}=-\frac{\partial \mathcal{P}^{L}}{\partial R_{j}}(\mathbf{r}(\mathbf{R}))=-\frac{1}{\nu_{j}} \frac{\partial \mathcal{P}^{L}}{\partial r_{j}}(\mathbf{r}), \quad j=-N, \ldots, N .
$$

We next derive the following relation between $f_{j}$ and the external conjugate force $\Phi_{j}$ :

$$
\begin{aligned}
f_{j} & =-\frac{\partial \mathcal{P}^{L}}{\partial z_{j}}(\mathbf{r})=-\frac{\partial \mathcal{P}^{L}}{\partial R_{j}}(\mathbf{r}(\mathbf{R})) \frac{\partial R_{j}}{\partial z_{j}}-\frac{\partial \mathcal{P}^{L}}{\partial R_{j-1}}(\mathbf{r}(\mathbf{R})) \frac{\partial R_{j-1}}{\partial z_{j}} \\
& =-\left(\Phi_{j}-\Phi_{j-1}\right), \quad j=-N, \ldots, N+1,
\end{aligned}
$$

where we set

$$
\Phi_{-N-1}=\Phi_{N+1}=0 .
$$

We can sum the external forces from the left of the chain and use the preceding equation (3.14) to obtain that

$$
\Phi_{j}=-\sum_{i=-N}^{j} f_{i}, \quad j=-N, \ldots, N+1 .
$$

If the external forces, $f_{j}$, are anti-symmetric, that is,

$$
f_{j+1}=-f_{-j}, \quad j=0, \ldots, N
$$

then we can conclude from (3.15) that the external conjugate force, $\Phi_{j}$, is symmetric about $j=0$, that is,

$$
\Phi_{j}=\Phi_{-j}, \quad j=-N-1, \ldots, N+1 .
$$

We note that the external forces, $f_{j}$, are anti-symmetric if the chain is subject only to tensile or compressive loads of equal magnitude, but opposite sign, at its ends. 


\subsection{Equilibrium equations}

We now derive equilibrium equations in terms of the symmetric internal conjugate force, $\hat{\psi}_{j}^{F}$, and discuss when solutions of these equilibrium equations correspond to solutions of $(\mathrm{F})$. The same is done for $\hat{\psi}_{j}^{E}$.

It follows from (3.5), (3.6), (3.15), and (3.14) that the force-based quasicontinuum equilibrium equations

$$
F_{j}^{Q C F}(\mathbf{r})+f_{j}=0, \quad j=-N, \ldots, N+1,
$$

are equivalent to

$$
\psi_{j}^{F}(\mathbf{r})=\Phi_{j}, \quad j=-N-1, \ldots, N+1 .
$$

We recall that $\Phi_{N+1}=0$ since we have assumed that the external forces satisfy the condition of no resultant force (3.12). Therefore, if $\mathbf{r}$ satisfies the force-based quasicontinuum equilibrium equations $(\mathrm{F})$, then $\psi_{N+1}(\mathbf{r})=0$, and by $(3.8)$ we have $\psi_{j}^{F}(\mathbf{r})=\hat{\psi}_{j}^{F}(\mathbf{r})$ for all $j=-N-1, \ldots, N+1$. Thus, we see that if $\mathbf{r}$ satisfies the force-based equilibrium equations $(\mathrm{F})$, then

$$
\hat{\psi}_{j}^{F}(\mathbf{r})=\Phi_{j}, \quad j=-N-1, \ldots, N+1 .
$$

We also recall that if $\mathbf{r}$ is symmetric, then

$$
\psi_{j}^{F}(\mathbf{r})=\hat{\psi}_{j}^{F}(\mathbf{r}), \quad j=-N-1, \ldots, N+1 .
$$

Hence, if $\mathbf{r}$ is a symmetric solution of

$$
\hat{\psi}_{j}^{F}(\mathbf{r})=\Phi_{j}, \quad j=-N-1, \ldots, N+1,
$$

then $\mathbf{r}$ is a solution of the force-based quasicontinuum equilibrium equations $(\mathrm{F})$. The equilibrium equations $(\mathrm{CF})$ are used in the following analysis and provide criteria for the existence of solutions to the equilibrium equations $(\mathrm{F})$.

If we sum the ghost force iteration equations

$$
F_{j}^{Q C E}\left(\mathbf{r}^{n+1}\right)+F_{j}^{G}\left(\mathbf{r}^{n}\right)+f_{j}=0, \quad j=-N, \ldots, N+1,
$$

then we also get the following equivalent corresponding iterative method in terms of the internal and external conjugate forces

$$
\psi_{j}^{E}\left(\mathbf{r}^{n+1}\right)+\psi_{j}^{G}\left(\mathbf{r}^{n}\right)=\Phi_{j}, \quad j=-N-1, \ldots, N+1 .
$$

We also have that if the sequence $\left\{\mathbf{r}^{n}\right\}$ satisfies the iteration equations (E), then the sequence $\left\{\mathbf{r}^{n}\right\}$ satisfies

$$
\hat{\psi}_{j}^{E}\left(\mathbf{r}^{n+1}\right)+\hat{\psi}_{j}^{G}\left(\mathbf{r}^{n}\right)=\Phi_{j}, \quad j=-N-1, \ldots, N+1 .
$$

We finally note that if the sequence $\left\{\mathbf{r}^{n}\right\}$ is a symmetric solution of $(\mathrm{CE})$, then the sequence $\left\{\mathbf{r}^{n}\right\}$ satisfies the ghost force iteration equations (E).

\section{ExistenCE OF SOLUTIONS TO THE FORCE-BASED QUASICONTINUUM SYSTEM}

In this section, we will give conditions on $\Phi=\left(\Phi_{-N}, \ldots, \Phi_{N}\right) \in \mathbb{R}^{2 N+1}$ such that there exists a unique solution $\mathbf{r}$ to

$$
\hat{\mathbf{\Psi}}^{F}(\mathbf{r})=\boldsymbol{\Phi}
$$

in a domain $\Omega \subset \mathbb{R}^{2 N+1}$, where $\hat{\Psi}^{F}(\mathbf{r})=\left(\hat{\psi}_{-N}^{F}(\mathbf{r}), \ldots, \hat{\psi}_{N}^{F}(\mathbf{r})\right) \in \mathbb{R}^{2 N+1}$ has the symmetric form given in (3.10). We note that we ignore $\hat{\psi}_{-N-1}^{F}, \hat{\psi}_{N+1}^{F}, \Phi_{-N-1}, \Phi_{N+1}$ in the above formulation since 
$\hat{\psi}_{-N-1}^{F}=\hat{\psi}_{N+1}^{F}=\Phi_{-N-1}=\Phi_{N+1}=0$. We conclude this section by showing that the unique symmetric solution $\mathbf{r}$ to (CF) for a symmetric $\boldsymbol{\Phi}$ is a solution of the force-based equilibrium equations (F).

For any uniform lattice spacing $\mathbf{a}=(a, \ldots, a) \in \mathbb{R}^{2 N+1}$, we have a corresponding uniform $\hat{\boldsymbol{\Psi}}^{F}(\mathbf{a})$. Under appropriate assumptions on the atomistic potential, $\phi(r)$, and the interatomic spacing, a, we will give an explicit neighborhood around $\mathbf{a}$ in which we have a unique solution to $(\mathrm{CF})$ for any external potential, $\boldsymbol{\Phi}$, in an explicit neighborhood of $\hat{\Psi}^{F}(\mathbf{a})$. We will show that these assumptions on the atomistic potential, $\phi(r)$, are satisfied by the Lennard-Jones potential

$$
\phi(r)=\frac{1}{r^{12}}-\frac{2}{r^{6}} .
$$

\subsection{Assumptions on the atomistic potential, $\phi(r)$}

We now give the assumptions on the atomistic potential, $\phi(r)$, that are required for our analysis. We will assume that $\phi(r) \in C^{3}((0, \infty))$, and we recall that $\eta(r)=\phi^{\prime}(r)$ and $\hat{\phi}(r)=\phi(r)+\phi(2 r)$. We define

$$
\hat{\eta}(r)=\hat{\phi}^{\prime}(r)
$$

so

$$
\hat{\eta}(r)=\phi^{\prime}(r)+2 \phi^{\prime}(2 r)=\eta(r)+2 \eta(2 r) .
$$

We assume that the atomistic potential, $\phi(r)$, satisfies the following properties that are graphically displayed in Figures 6 and 7:

$$
\begin{aligned}
& \eta^{\prime}(r)>0 \text { for } 0<r<\tilde{r}_{1} \text { and } \eta^{\prime}(r)<0 \text { for } r>\tilde{r}_{1}, \\
& \eta^{\prime \prime}(r)<0 \text { for } 0<r<\tilde{r}_{2} \text { and } \eta^{\prime \prime}(r)>0 \text { for } r>\tilde{r}_{2}, \\
& \hat{\eta}(r)<0 \text { for } 0<r<a_{0} \text { and } \hat{\eta}(r)>0 \text { for } r>a_{0}, \\
& \hat{\eta}^{\prime}(r)>0 \text { for } 0<r<a_{1} \text { and } \hat{\eta}^{\prime}(r)<0 \text { for } r>a_{1}, \\
& 0<a_{0}<\tilde{r}_{1}<\tilde{r}_{2}<2 a_{0}, \\
& a_{0}<a_{1} .
\end{aligned}
$$

We note that $a_{0}$ is the equilibrium bond length of a uniform chain, and $a_{1}$ is the bond length of a uniform chain at the load limit. It follows from (4.4) that $F_{j}^{Q C F}\left(\mathbf{a}_{0}\right)=0$ for $j=-N, \ldots, N+1$ and $\hat{\Psi}^{F}\left(\mathbf{a}_{0}\right)=\mathbf{0}$ where $\mathbf{a}_{0}=\left(a_{0}, \ldots, a_{0}\right) \in \mathbb{R}^{2 N+1}$.

The local quasicontinuum approximation is simply the case where all of the representative atoms are continuum, giving a decoupled system

$$
\hat{\mathbf{\Psi}}^{L}(\mathbf{r})=\mathbf{\Phi}
$$

where $\hat{\Psi}^{L}(\mathbf{r})=\left(\hat{\psi}_{-N}^{L}(\mathbf{r}), \ldots, \hat{\psi}_{N}^{L}(\mathbf{r})\right)$ is defined by

$$
\hat{\psi}_{i}^{L}(\mathbf{r})=\hat{\eta}\left(r_{i}\right), \quad i=-N, \ldots, N .
$$

We thus have that

$$
D_{j} \hat{\psi}_{i}^{L}(\mathbf{r})=\hat{\eta}^{\prime}\left(r_{i}\right) \delta_{i j},
$$

and we see that the local quasicontinuum approximation is unstable when $r_{i}>a_{1}$ for some $i=-N, \ldots, N$.

\subsection{Existence and uniqueness by the inverse function theorem}

While the main theorem of this section gives explicit conditions on $\boldsymbol{\Phi}$ for which $\hat{\mathbf{\Psi}}^{F}(\mathbf{r})=\boldsymbol{\Phi}$ is solvable, we begin by showing that the inverse function theorem [4] can be used to show that $\hat{\mathbf{\Psi}}^{F}(\mathbf{r})$ is bijective 

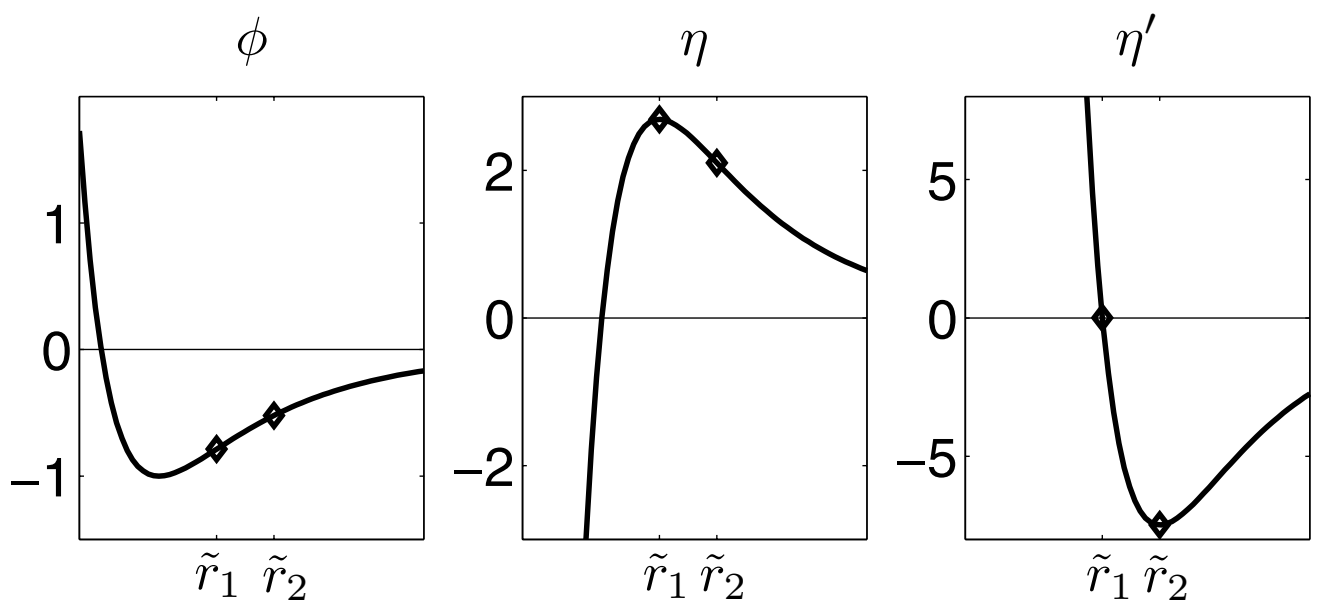

FIGURE 6. The Lennard-Jones potential (4.1) demonstrates the prototypical behavior of $\phi(r)$ and its derivatives, $\eta(r)=\phi^{\prime}(r)$ and $\eta^{\prime}(r)=\phi^{\prime \prime}(r)$.
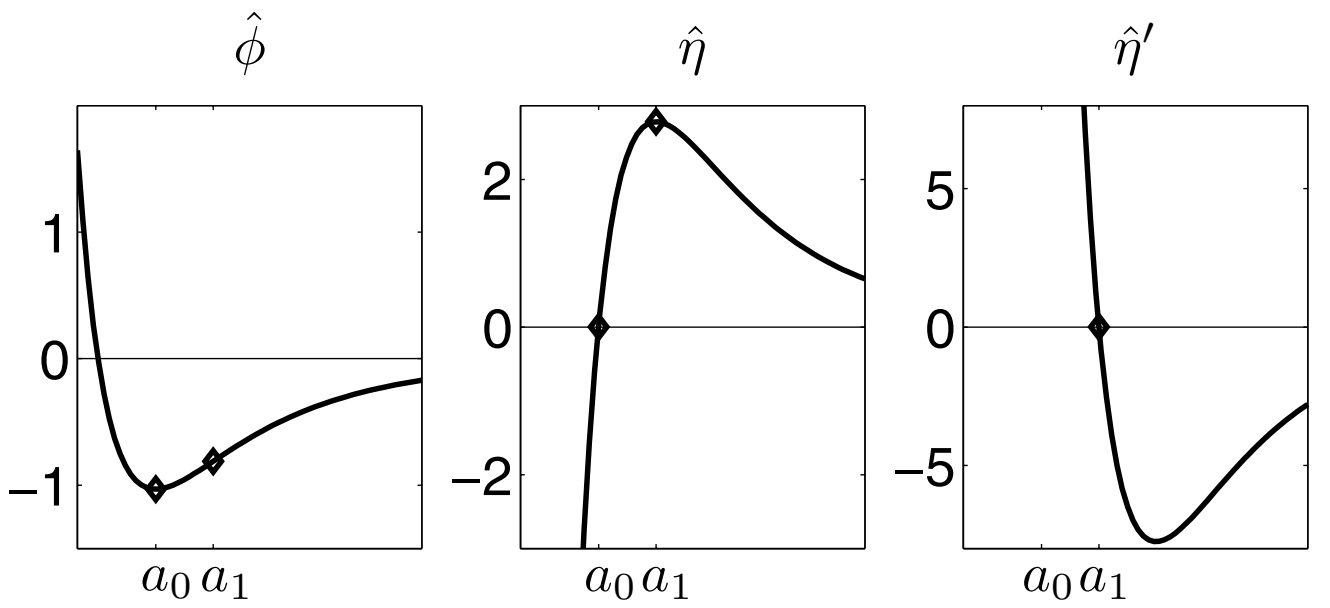

Figure 7. The energy density, $\hat{\phi}(r)$, for the Lennard-Jones potential (4.1) and its derivatives, $\hat{\eta}(r)=\hat{\phi}^{\prime}(r)$ and $\hat{\eta}^{\prime}(r)=\hat{\phi}^{\prime \prime}(r)$.

in a neighborhood of $\mathbf{r}=\mathbf{a}_{0}$. Therefore, we must analyze the invertibility of

$$
D_{j} \hat{\psi}_{i}^{F}(\mathbf{r})=\left\{\begin{array}{rlrl}
{\left[\eta^{\prime}\left(r_{i}\right)+4 \eta^{\prime}\left(2 r_{i}\right)\right] \delta_{i j},} & & -N \leq i \leq-K, \\
& \left.\eta^{\prime}\left(r_{i}\right)+\eta^{\prime}\left(r_{i}+r_{i-1}\right)+\eta^{\prime}\left(r_{i}+r_{i+1}\right)\right] \delta_{i j} & \\
& \quad+\eta^{\prime}\left(r_{i}+r_{i-1}\right) \delta_{i-1 j}+\eta^{\prime}\left(r_{i}+r_{i+1}\right) \delta_{i+1 j} & \\
& \quad+\left[4 \eta^{\prime}\left(2 r_{K}\right)-\eta^{\prime}\left(r_{K}+r_{K-1}\right)-\eta^{\prime}\left(r_{K}+r_{K+1}\right)\right] \delta_{K j} j & \\
& -\eta^{\prime}\left(r_{K}+r_{K-1}\right) \delta_{K-1 j}-\eta^{\prime}\left(r_{K}+r_{K+1}\right) \delta_{K+1 j}, & & -K+1 \leq i \leq K-1, \\
& {\left[\eta^{\prime}\left(r_{i}\right)+4 \eta^{\prime}\left(2 r_{i}\right)\right] \delta_{i j},} & & K \leq i \leq N .
\end{array}\right.
$$


Lemma 4.1. If $\eta^{\prime}\left(a_{0}\right)+8 \eta^{\prime}\left(2 a_{0}\right)>0$, then $\hat{\mathbf{\Psi}}^{F}(\mathbf{r})$ is bijective in a neighborhood of $\mathbf{a}_{0}$.

Proof. We will show that $D \hat{\mathbf{\Psi}}^{F}\left(\mathbf{a}_{0}\right)$ (where $\left.\left(D \hat{\boldsymbol{\Psi}}^{F}\right)_{i j}=D_{j} \hat{\psi}_{i}^{F}\right)$ is nonsingular by demonstrating that it is strictly diagonal dominant [21] (with positive diagonal), that is,

$$
D_{i} \hat{\psi}_{i}^{F}\left(\mathbf{a}_{0}\right)>\sum_{j \neq i}\left|D_{j} \hat{\psi}_{i}^{F}\left(\mathbf{a}_{0}\right)\right|, \quad i=-N, \ldots, N .
$$

Looking at the rows of $D \hat{\Psi}^{F}\left(\mathbf{a}_{0}\right)$, all of the nearest-neighbor terms are on the diagonal. If we collect all of the next-neighbor terms on the right hand side of the above inequality, we find that it is sufficient to show that $\eta^{\prime}\left(a_{0}\right)>4\left|\eta^{\prime}\left(2 a_{0}\right)\right|$ for rows $i=-N, \ldots,-K$ and $i=K, \ldots, N$ and $\eta^{\prime}\left(a_{0}\right)>8\left|\eta^{\prime}\left(2 a_{0}\right)\right|$ for rows $i=-K+1, \ldots, K-1$. Since $2 a_{0}>\tilde{r}_{1}$ by assumption (4.6), we have that $\eta^{\prime}\left(2 a_{0}\right)<0$ by (4.2). Thus, the condition $\eta^{\prime}\left(a_{0}\right)+8 \eta^{\prime}\left(2 a_{0}\right)>0$ implies the strict diagonal dominance of (4.9). Therefore, we have that $D \hat{\mathbf{\Psi}}^{F}\left(\mathbf{a}_{0}\right)$ is nonsingular, and so by the inverse function theorem there exists a neighborhood of $\mathbf{a}_{0}$ in which $\hat{\mathbf{\Psi}}^{F}(\mathbf{r})$ is bijective.

\subsection{Existence and uniqueness for general external forces}

In the theorems of this section and the next, we will use the continuation method [1] to find explicit conditions under which $\hat{\boldsymbol{\Psi}}^{F}(\mathbf{r})=\boldsymbol{\Phi}$ has a unique solution. The idea of the continuation method is to start with the uncoupled local quasicontinuum system, construct a homotopy transformation from the local quasicontinuum system to the force-based quasicontinuum system, and show that existence and uniqueness persists through the transformation. We use the following well-known lemma to show that unique solvability of the local quasicontinuum system implies existence and uniqueness of a solution to the force-based quasicontinuum system [4].

Lemma 4.2. Let $\Omega \subset \mathbb{R}^{2 N+1}$ be an open, bounded set. Suppose that $\mathbf{f}, \mathbf{g} \in C^{1}\left(\bar{\Omega} ; \mathbb{R}^{2 N+1}\right)$ and the homotopy $\mathbf{h}(\mathbf{r}, t)=(1-t) \mathbf{f}(\mathbf{r})+t \mathbf{g}(\mathbf{r})$ for $t \in[0,1]$ satisfies

(1) $\mathbf{f}(\mathbf{r})=0$ has a unique solution in $\Omega$,

(2) $\operatorname{det} D_{\mathbf{r}} \mathbf{h}(\mathbf{r}, t) \neq 0$ in $\Omega \times[0,1]$,

(3) $\mathbf{h}(\mathbf{r}, t) \neq 0$ for every $(\mathbf{r}, t) \in \partial \Omega \times[0,1]$.

Then there is a unique solution $\mathbf{r} \in \Omega$ satisfying $\mathbf{g}(\mathbf{r})=0$.

Proof. Since $\operatorname{det} D_{\mathbf{r}} \mathbf{h}(\mathbf{r}, t) \neq 0$ for all $(\mathbf{r}, t) \in \Omega \times[0,1]$, we know by the implicit function theorem [4] that the solution of $h(\mathbf{r}, t)=0$ in a neighborhood of a particular solution $\left(\mathbf{r}_{*}, t_{*}\right)$ satisfying $h\left(\mathbf{r}_{*}, t_{*}\right)=0$ can be written as a function $\mathbf{r}(t)$ in a neighborhood of $\left(\mathbf{r}_{*}, t_{*}\right)$. Thus, by compactness, the curve can be continued until it leaves the region $\bar{\Omega} \times[0,1]$. Since we have assumed that $\mathbf{h}(\mathbf{r}, t) \neq 0$ on $\partial \Omega \times[0,1]$, there is a one-to-one correspondence between solutions of $\mathbf{f}(\mathbf{r})=0$ and solutions of $\mathbf{g}(\mathbf{r})=0$. Therefore, the existence of a unique solution of $\mathbf{f}(\mathbf{r})=0$ for $\mathbf{r} \in \Omega$ implies that $\mathbf{g}(\mathbf{r})=0$ has a unique solution for $\mathbf{r} \in \Omega$.

We define the homotopy

$$
\mathbf{h}(\mathbf{r}, t)=(1-t)\left[\hat{\mathbf{\Psi}}^{L}(\mathbf{r})-\mathbf{\Phi}\right]+t\left[\hat{\mathbf{\Psi}}^{F}(\mathbf{r})-\mathbf{\Phi}\right], \quad t \in[0,1]
$$

where $\hat{\boldsymbol{\Psi}}^{L}(\mathbf{r})$ is the local quasicontinuum system (4.8). The next lemma uses the properties of the local quasicontinuum system to simplify the hypothesis for the previous lemma.

Lemma 4.3. Let $r_{L}$ and $r_{U}$ satisfy $0<r_{L}<r_{U}<a_{1}$, and set $\Omega=\left(r_{L}, r_{U}\right)^{2 N+1}$. Suppose that for $i=$ $-N, \ldots, N$ we have that

$$
\begin{aligned}
& h_{i}(\mathbf{r}, t)>0 \text { if } r_{i}=r_{U} \text { and } \mathbf{r} \in \partial \Omega, t \in[0,1], \\
& h_{i}(\mathbf{r}, t)<0 \text { if } r_{i}=r_{L} \text { and } \mathbf{r} \in \partial \Omega, t \in[0,1]
\end{aligned}
$$


If $D \hat{\Psi}^{F}(\mathbf{r})$ is strictly diagonally dominant for all $\mathbf{r} \in \Omega$, then there exists a unique solution in $\Omega$ to the system of equations $\hat{\mathbf{\Psi}}^{F}(\mathbf{r})=\boldsymbol{\Phi}$.

Proof. We show that the above conditions are enough to satisfy the hypotheses of Lemma 4.2. We first show that $\hat{\boldsymbol{\Psi}}^{L}(\mathbf{r})=\boldsymbol{\Phi}$ has a unique solution in $\Omega$. Since the system is decoupled, we need only demonstrate that the scalar equations

$$
\eta\left(r_{j}\right)+2 \eta\left(2 r_{j}\right)=\Phi_{j}, \quad j=-N, \ldots, N,
$$

have a unique solution for $r_{L}<r_{j}<r_{U}$. We have from the hypothesis (4.10) on $h_{i}(\mathbf{r}, t)$ at $t=0$ that $\eta\left(r_{L}\right)+2 \eta\left(2 r_{L}\right)-\Phi_{j}<0$ and $\eta\left(r_{U}\right)+2 \eta\left(2 r_{U}\right)-\Phi_{j}>0$. Hence, a solution $r_{j}$ to (4.11) exists by the intermediate value theorem. Since $\hat{\eta}(r)=\eta(r)+2 \eta(2 r)$ is increasing for $0<r<r_{U}<a_{1}$ by (4.5), the solution to (4.11) must be unique.

The hypothesis (4.10) implies that $\mathbf{h}(\mathbf{r}, t) \neq 0$ for every $(\mathbf{r}, t) \in \partial \Omega \times[0,1]$. Finally, to show that $\operatorname{det} D_{\mathbf{r}} \mathbf{h}(\mathbf{r}, t) \neq 0$ for all $(\mathbf{r}, t) \in \Omega \times[0,1]$ it is sufficient to demonstrate the strict diagonal dominance of $D_{\mathbf{r}} \mathbf{h}(\mathbf{r}, t)$ for all $(\mathbf{r}, t) \in \Omega \times[0,1]$.

Now

$$
D_{j} h_{i}(\mathbf{r}, t)=(1-t) D_{j} \hat{\psi}_{i}^{L}(\mathbf{r})+t D_{j} \hat{\psi}_{i}^{F}(\mathbf{r})
$$

and

$$
D_{j} \hat{\psi}_{i}^{L}(\mathbf{r})=\hat{\eta}^{\prime}\left(r_{i}\right) \delta_{i j} .
$$

Since $0<r_{i}<a_{1}$, we have by (4.5) that $\hat{\eta}^{\prime}\left(r_{i}\right)>0$. Thus, we have that $D_{j} \hat{\psi}_{i}^{L}(\mathbf{r})$ is strictly diagonal dominant (with positive diagonal). We can then conclude that $D_{j} h_{i}(\mathbf{r}, t)$ is strictly diagonal dominant (with positive diagonal) if $D_{j} \hat{\psi}_{i}^{F}(\mathbf{r})$ is strictly diagonal dominant (with positive diagonal) since $D_{j} h_{i}(\mathbf{r}, t)$ is then the sum of two strictly diagonal dominant matrices (with positive diagonal). Thus, by Lemma 4.2, the equation $\hat{\mathbf{\Psi}}^{F}(\mathbf{r})-\boldsymbol{\Phi}=0$ has a unique solution in $\Omega$.

We next turn to giving results that allow the calculation of an explicit neighborhood of a for which $\hat{\Psi}^{F}(\mathbf{r})$ is bijective. We first give a condition on $0<r_{L}<r_{U}$ such that $D \hat{\mathbf{\Psi}}^{F}(\mathbf{r})$ is strictly diagonally dominant for all $\mathbf{r} \in \Omega$ where $\Omega=\left(r_{L}, r_{U}\right)^{2 N+1}$.

Lemma 4.4. Suppose that $r_{L}$ and $r_{U}$ satisfy

$$
\begin{aligned}
& \frac{\tilde{r}_{2}}{2}<r_{L}<r_{U}, \\
& \eta^{\prime}\left(r_{U}\right)+12 \eta^{\prime}\left(2 r_{L}\right) \geq 0 .
\end{aligned}
$$

Then $r_{U}<a_{1}$, and $D \hat{\mathbf{\Psi}}^{F}(\mathbf{r})$ is strictly diagonally dominant for all $\mathbf{r} \in \Omega$ where $\Omega=\left(r_{L}, r_{U}\right)^{2 N+1}$.

Proof. First, we note that since $r_{L}>\frac{\tilde{r}_{2}}{2}$, (4.3) implies that $12 \eta^{\prime}\left(2 r_{L}\right)<12 \eta^{\prime}\left(2 r_{U}\right)$. Also, (4.3) gives that the next-neighbor terms are negative, so that $\eta^{\prime}\left(r_{U}\right)+4 \eta^{\prime}\left(2 r_{U}\right)>\eta^{\prime}\left(r_{U}\right)+12 \eta^{\prime}\left(2 r_{U}\right) \geq 0$. Therefore, by (4.6) we can conclude that $r_{U}<a_{1}$.

We can obtain from (4.9) by summing all of the next-nearest-neighbor terms in each row that $D \hat{\mathbf{\Psi}}^{F}(\mathbf{r})$ is strictly diagonally dominant if

$$
\eta^{\prime}(r)>12\left|\eta^{\prime}(2 s)\right| \quad \text { for all } r, s \in\left(r_{L}, r_{U}\right) .
$$

(We note that the hypothesis of Lem. 4.1 required the weaker condition $\eta^{\prime}\left(a_{0}\right)>8\left|\eta^{\prime}\left(2 a_{0}\right)\right|$ since we were able to utilize the cancellation of terms in the expression

$$
D_{K} \hat{\psi}_{i}^{F}(\mathbf{r})=4 \eta^{\prime}\left(2 r_{K}\right)-\eta^{\prime}\left(r_{K}+r_{K-1}\right)-\eta^{\prime}\left(r_{K}+r_{K+1}\right), \quad i=-K+1, \ldots, K-1,
$$

when it was evaluated at $\mathbf{r}=\mathbf{a}_{0}$ to obtain that $D_{K} \hat{\psi}_{i}^{F}\left(\mathbf{a}_{0}\right)=2 \eta^{\prime}\left(2 a_{0}\right)$ for $i=-K+1, \ldots, K-1$.) 
We have by (4.2) and (4.3) that

$$
\eta^{\prime}(r)>0 \text { and } \eta^{\prime}(r) \text { is decreasing for } r<\tilde{r}_{1},
$$

and we have that

$$
\eta^{\prime}(2 s)<0 \text { and } \eta^{\prime}(2 s) \text { is increasing for } s>\frac{\tilde{r}_{2}}{2} \text {. }
$$

It follows from (4.14) that to prove strict diagonal dominance it is sufficient to show that

$$
\eta^{\prime}(r)+12 \eta^{\prime}(2 s)>0 \quad \text { for all } r, s \in\left(r_{L}, r_{U}\right) .
$$

We have from (4.13) and (4.14) that the above condition follows from the hypothesis (4.12).

Theorem 4.1. Suppose that $r_{L}$ and $r_{U}$ satisfy

$$
\begin{aligned}
& \frac{\tilde{r}_{2}}{2}<r_{L}<r_{U}, \\
& \eta^{\prime}\left(r_{U}\right)+12 \eta^{\prime}\left(2 r_{L}\right) \geq 0 .
\end{aligned}
$$

If

$$
\eta\left(r_{L}\right)+4 \eta\left(2 r_{L}\right)-2 \eta\left(2 r_{U}\right)<\Phi_{j}<\eta\left(r_{U}\right)+4 \eta\left(2 r_{U}\right)-2 \eta\left(2 r_{L}\right), \quad j=-N, \ldots, N,
$$

then there is a unique solution in $\Omega=\left(r_{L}, r_{U}\right)^{2 N+1}$ to the system of equations $\hat{\mathbf{\Psi}}^{F}(\mathbf{r})=\boldsymbol{\Phi}$.

Remark 4.1. The restriction on $\Phi_{j}$ corresponds to a restriction on the cumulative effect of the external forces $f_{j}$. The forces need not be small or restricted to the regime of linear elasticity, and they can be distributed throughout the chain or focused on only a single pair of representative atoms as in the case of a chain in tension. However, the condition does put an absolute bound on the magnitude by requiring that their total effect does not exceed the load limit of the chain.

Proof. We have by Lemma 4.4 that $D \hat{\mathbf{\Psi}}^{F}(\mathbf{r})$ is strictly diagonally dominant for all $\mathbf{r} \in \Omega$. By Lemma 4.2, we need only show that

$$
\begin{aligned}
& h_{i}(\mathbf{r}, t)>0 \text { if } r_{i}=r_{U} \text { and } \mathbf{r} \in \partial \Omega, t \in[0,1], \\
& h_{i}(\mathbf{r}, t)<0 \text { if } r_{i}=r_{L} \text { and } \mathbf{r} \in \partial \Omega, t \in[0,1] .
\end{aligned}
$$

If we look at the entries of $\hat{\Psi}^{F}(\mathbf{r})$, we see that there is always one nearest-neighbor term and at most four positive and two negative next-nearest-neighbor terms (3.10). We also note that $2 r_{L}>\tilde{r}_{2}>\tilde{r}_{1}$ by (4.15) and (4.6), so we have by (4.2) that $\eta(2 r)$ is deceasing for $r \geq r_{L}$ and

$$
2 \eta\left(2 r_{L}\right)-2 \eta\left(2 r_{U}\right)>0 .
$$

We can thus estimate $\mathbf{h}(\mathbf{r}, t)$ on the boundary to get

$$
\begin{aligned}
\min _{\mathbf{r} \in \partial \Omega, r_{j}=r_{U}} h_{j}(\mathbf{r}, t) & =\min _{\mathbf{r} \in \partial \Omega, r_{j}=r_{U}}(1-t)\left[\hat{\psi}_{j}^{L}(\mathbf{r})-\Phi_{j}\right]+t\left[\hat{\psi}_{j}^{F}(\mathbf{r})-\Phi_{j}\right] \\
& \geq(1-t)\left[\eta\left(r_{U}\right)+2 \eta\left(2 r_{U}\right)-\Phi_{j}\right]+t\left[\eta\left(r_{U}\right)+4 \eta\left(2 r_{U}\right)-2 \eta\left(2 r_{L}\right)-\Phi_{j}\right] \\
& =(1-t)\left[2 \eta\left(2 r_{L}\right)-2 \eta\left(2 r_{U}\right)\right]+\left[\eta\left(r_{U}\right)+4 \eta\left(2 r_{U}\right)-2 \eta\left(2 r_{L}\right)\right]-\Phi_{j}>0, \\
\max _{\mathbf{r} \in \partial \Omega, r_{j}=r_{L}} h_{j}(\mathbf{r}, t) & =\max _{\mathbf{r} \in \partial \Omega, r_{j}=r_{L}}(1-t)\left[\hat{\psi}_{j}^{L}(\mathbf{r})-\Phi_{j}\right]+t\left[\hat{\psi}_{j}^{F}(\mathbf{r})-\Phi_{j}\right] \\
& \leq(1-t)\left[\eta\left(r_{L}\right)+2 \eta\left(2 r_{L}\right)-\Phi_{j}\right]+t\left[\eta\left(r_{L}\right)+4 \eta\left(2 r_{L}\right)-2 \eta\left(2 r_{U}\right)-\Phi_{j}\right] \\
& =(1-t)\left[2 \eta\left(2 r_{U}\right)-2 \eta\left(2 r_{L}\right)\right]+\left[\eta\left(r_{L}\right)+4 \eta\left(2 r_{L}\right)-2 \eta\left(2 r_{U}\right)\right]-\Phi_{j}<0 .
\end{aligned}
$$

Therefore, by Lemma 4.3 , we have a unique solution to $\hat{\mathbf{\Psi}}^{F}(\mathbf{r})=\mathbf{\Phi}$ in $\Omega$. 
We now consider the case in which the external forces, $f_{j}$, are anti-symmetric (3.16), so that the external potential $\Phi_{j}$ is symmetric (3.17). We then have that the solution $\mathbf{r}$ to $\hat{\boldsymbol{\Psi}}^{F}(\mathbf{r})=\mathbf{\Phi}$ obtained in the proof of Theorem 4.1 is symmetric, and will also solve the equilibrium equations (F).

Corollary 4.1. Suppose that $r_{L}$ and $r_{U}$ satisfy

$$
\begin{aligned}
& \frac{\tilde{r}_{2}}{2}<r_{L}<r_{U}, \\
& \eta^{\prime}\left(r_{U}\right)+12 \eta^{\prime}\left(2 r_{L}\right) \geq 0,
\end{aligned}
$$

and that the external forces, $f_{j}$, are anti-symmetric (3.16). If

$$
\eta\left(r_{L}\right)+4 \eta\left(2 r_{L}\right)-2 \eta\left(2 r_{U}\right)<\Phi_{j}<\eta\left(r_{U}\right)+4 \eta\left(2 r_{U}\right)-2 \eta\left(2 r_{L}\right), \quad j=-N, \ldots, N,
$$

then there is a unique symmetric solution in $\Omega=\left(r_{L}, r_{U}\right)^{2 N+1}$ to the equilibrium equations

$$
F_{j}^{Q C F}(\mathbf{r})+f_{j}=0, \quad j=-N, \ldots, N+1 .
$$

Proof. We first note that since the external forces, $f_{j}$, are anti-symmetric, the external potential $\mathbf{\Phi}$ is symmetric. We consider the solution $\mathbf{r}(t)$ to the homotopy continuation

$$
\mathbf{h}(\mathbf{r}(t), t)=(1-t)\left[\hat{\mathbf{\Psi}}^{L}(\mathbf{r}(t))-\mathbf{\Phi}\right]+t\left[\hat{\mathbf{\Psi}}^{F}(\mathbf{r}(t))-\mathbf{\Phi}\right], \quad t \in[0,1]
$$

that we have from Lemma 4.2. The unique solution to the decoupled local quasicontinuum system $\hat{\boldsymbol{\Psi}}^{L}(\mathbf{r})=\boldsymbol{\Phi}$ is symmetric, therefore $\mathbf{r}(0)$ is symmetric. We obtain by differentiating (4.18) that

$$
\nabla_{\mathbf{r}} \mathbf{h}(\mathbf{r}(t), t) \mathbf{r}_{t}(t)+\mathbf{h}_{t}(\mathbf{r}(t), t)=0, \quad t \in[0,1],
$$

where

$$
\nabla_{\mathbf{r}} \mathbf{h}(\mathbf{r}(t), t)=(1-t) \nabla_{\mathbf{r}} \hat{\Psi}^{L}(\mathbf{r}(t))+t \nabla_{\mathbf{r}} \hat{\Psi}^{F}(\mathbf{r}(t))
$$

and

$$
\mathbf{h}_{t}(\mathbf{r}(t), t)=\hat{\mathbf{\Psi}}^{F}(\mathbf{r}(t))-\hat{\mathbf{\Psi}}^{L}(\mathbf{r}(t)) .
$$

All terms above are symmetric whenever $\mathbf{r}(t)$ is, and therefore the symmetry of $\mathbf{r}(0)$ implies that $\mathbf{r}_{t}(t)$ and $\mathbf{r}(t)$ are symmetric in the whole interval $[0,1]$, and in particular $\mathbf{r}(1)$ is symmetric. The proof that $\mathbf{r}$ is symmetric is completed by observing that $\mathbf{r}(1)$ is the unique solution to $\hat{\Psi}^{F}(\mathbf{r})=\mathbf{\Phi}$.

Since the unique solution $\mathbf{r}$ to $\hat{\Psi}^{F}(\mathbf{r})=\boldsymbol{\Phi}$ is symmetric, we can conclude that $\mathbf{r}$ is the unique symmetric solution to the force-based equilibrium equations

$$
F_{j}^{Q C F}(\mathbf{r})+f_{j}=0, \quad j=-N, \ldots, N+1 .
$$

Remark 4.2. The assumption of anti-symmetric external forces allowed us to replace the force-based equilibrium equations $(\mathrm{F})$ with the symmetrized conjugate force equilibrium equations $(\mathrm{CF})$ since we obtain a symmetric solution. For more general external forces, our argument requires that there is no resultant internal force on the chain, that is, that (3.8) holds, and we do not see how to ensure this in general without putting some restriction on the form of $f_{j}$ in addition to the usual requirement of no resultant external force (3.12). It is likely that a more involved proof can give the existence of solutions to the force-based equilibrium equations (F) under weaker assumptions on the external forces $f_{j}$.

We now apply Corollary 4.1 to the Lennard-Jones potential (4.1). 
Corollary 4.2. We assume that the external forces, $f_{j}$, are anti-symmetric (3.16). For any $\frac{\tilde{r}_{2}}{2}<r_{U}<a_{1}$, let

$$
r_{L}=\max \left(\frac{\tilde{r}_{2}}{2},\left(\frac{63}{16 \eta^{\prime}\left(r_{U}\right)}\right)^{\frac{1}{8}}\right) \text {. }
$$

If $r_{L}<r_{U}$, then the equilibrium equations

$$
F_{j}^{Q C F}(\mathbf{r})+f_{j}=0, \quad j=-N, \ldots, N+1
$$

have a unique symmetric solution $\mathbf{r}$ in $\Omega=\left(r_{L}, r_{U}\right)^{2 N+1}$ whenever

$$
\eta\left(r_{L}\right)+4 \eta\left(2 r_{L}\right)-2 \eta\left(2 r_{U}\right)<\Phi_{j}<\eta\left(r_{U}\right)+4 \eta\left(2 r_{U}\right)-2 \eta\left(2 r_{L}\right), \quad j=-N, \ldots, N .
$$

Proof. To prove this corollary using Theorem 4.1, we need to show that (4.16) holds. Evaluating (4.16) for the Lennard-Jones potential (4.1), we get

$$
\begin{aligned}
\eta^{\prime}\left(r_{U}\right)+12 \eta^{\prime}\left(2 r_{L}\right) & =\eta^{\prime}\left(r_{U}\right)+12\left[\frac{156}{2^{14} r_{L}^{14}}-\frac{84}{2^{8} r_{L}^{8}}\right] \\
& >\eta^{\prime}\left(r_{U}\right)-\frac{63}{16 r_{L}^{8}}
\end{aligned}
$$

Therefore, (4.16) holds if $r_{L} \geq\left(\frac{63}{16 \eta^{\prime}\left(r_{U}\right)}\right)^{1 / 8}$.

After solving for $r_{L}$ in (4.19), we have to check the additional hypothesis (4.15) that $r_{L}<r_{U}$, as it is not true for every $r_{U} \in\left(\hat{r}_{2} / 2, a_{1}\right)$. However, this is not a very restrictive assumption since it is true for all $r_{U}<1.1003$, whereas $a_{1}=1.1059$. The lower end for the interval is $\frac{\tilde{r}_{2}}{2}=0.6085$.

Using Corollary 4.2, we now find a symmetric region about $\mathbf{\Phi}=0$. Solving numerically, if we take $r_{L}=0.9700$ and $r_{U}=1.0883$, we can conclude that for any $\boldsymbol{\Phi}$ satisfying $-2.62<\Phi<2.62$, we can uniquely solve the force-based quasicontinuum equilibrium equations (F). For the Lennard-Jones potential $\hat{\eta}\left(a_{1}\right)=2.781$, so this symmetric region extends quite close to the load limit.

Remark 4.3. The techniques of this section can be applied to the analysis of the fully atomistic model or the constrained atomistic quasicontinuum approximation, and the analysis will be simplified as there are no non-local conjugate forces. In both cases, the continuation from the local quasicontinuum approximation can be used.

We also note that the interfacial terms satisfy the convexity condition

$$
\mathcal{S}_{j}\left(r_{j-1}, r_{j}\right)=-\frac{1}{2} \phi\left(2 r_{j-1}\right)+\phi\left(r_{j-1}+r_{j}\right)-\frac{1}{2} \phi\left(2 r_{j}\right)>0, \quad j=-N+1, \ldots, N,
$$

for $\mathbf{r}$ in the region defined by (4.15) in Theorem 4.1 and Corollary 4.1 since (4.14) holds in this region.

\section{Convergence of the Ghost Force iteration}

We now give a similar analysis for the iterative equations (CE) which in vector form are

$$
\hat{\mathbf{\Psi}}^{E}\left(\mathbf{r}^{n+1}\right)+\hat{\mathbf{\Psi}}^{G}\left(\mathbf{r}^{n}\right)=\boldsymbol{\Phi}
$$

where $\hat{\mathbf{\Psi}}^{E}(\mathbf{r})=\left(\hat{\psi}_{-N}^{E}(\mathbf{r}), \ldots, \hat{\psi}_{N}^{E}(\mathbf{r})\right) \in \mathbb{R}^{2 N+1}$ has the symmetric form given in $(3.4)$, and $\hat{\boldsymbol{\Psi}}^{G}(\mathbf{r})=$ $\left(\hat{\psi}_{-N}^{G}(\mathbf{r}), \ldots, \hat{\psi}_{N}^{G}(\mathbf{r})\right) \in \mathbb{R}^{2 N+1}$ has the symmetric form given in (3.11). We also note that we can ignore 
$\hat{\psi}_{-N-1}^{E}, \hat{\psi}_{N+1}^{E}, \hat{\psi}_{-N-1}^{G}, \hat{\psi}_{N+1}^{G}$ and $\Phi_{-N-1}, \Phi_{N+1}$ in the above formulation since

$$
\hat{\psi}_{-N-1}^{E}=\hat{\psi}_{N+1}^{E}=\hat{\psi}_{-N-1}^{G}=\hat{\psi}_{N+1}^{G}=\Phi_{-N-1}=\Phi_{N+1}=0 .
$$

We will determine $\Omega \subset \mathbb{R}^{2 N+1}$ and $D \subset \mathbb{R}^{2 N+1}$ such that there is a unique $\mathbf{r}^{n+1} \in \Omega$ that satisfies (CE) whenever $\mathbf{r}^{n} \in \Omega$ and $\boldsymbol{\Phi} \in D$, and we will show that the induced mapping from $\mathbf{r}^{n}$ to $\mathbf{r}^{n+1}$ is a contraction mapping.

First, we need to compute $D \hat{\Psi}^{E}(\mathbf{r})$ and $D \hat{\Psi}^{G}(\mathbf{r})$. The following expressions are rather complex, but all we will need to know about them is the number of first and second neighbor terms in each row. We have

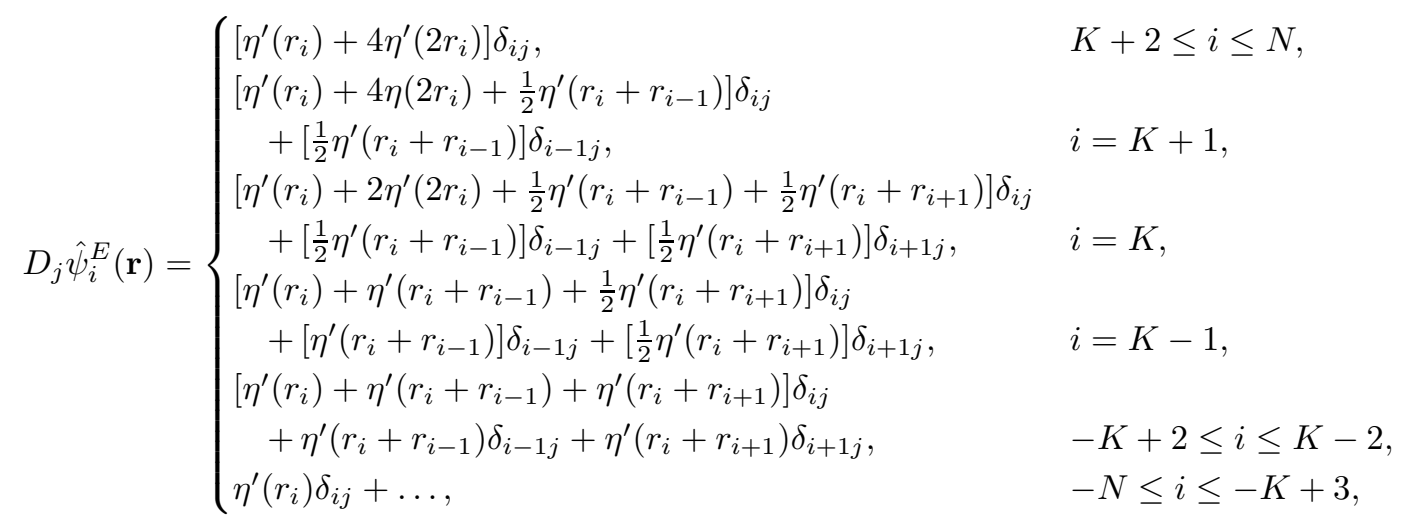

and



We recall that the maximum norm for $\mathbf{r} \in \mathbb{R}^{2 N+1}$ is given by

$$
\|\mathbf{r}\|_{\infty}=\max _{i=-N, \ldots, N}\left|r_{i}\right| .
$$

Theorem 5.1. Suppose that $r_{L}$ and $r_{U}$ satisfy

$$
\begin{aligned}
& \frac{\hat{r}_{2}}{2}<r_{L}<r_{U}, \\
& \eta^{\prime}\left(r_{U}\right)+13 \eta^{\prime}\left(2 r_{L}\right)>0,
\end{aligned}
$$

and that $\mathbf{\Phi}$ satisfies

$$
\eta\left(r_{L}\right)+4 \eta\left(2 r_{L}\right)-2 \eta\left(2 r_{U}\right)<\Phi_{j}<\eta\left(r_{U}\right)+4 \eta\left(2 r_{U}\right)-2 \eta\left(2 r_{L}\right), \quad j=-N, \ldots, N .
$$

Then for every $\mathbf{r}^{n} \in \Omega=\left(r_{L}, r_{U}\right)^{2 N+1}$ there is a unique $\mathbf{r}^{n+1} \in \Omega$ such that

$$
\hat{\mathbf{\Psi}}^{E}\left(\mathbf{r}^{n+1}\right)+\hat{\boldsymbol{\Psi}}^{G}\left(\mathbf{r}^{n}\right)=\mathbf{\Phi} .
$$


We also have that the induced mapping $\mathbf{r}^{n} \rightarrow \mathbf{r}^{n+1}$ is a contraction and satisfies the inequality

$$
\left\|\mathbf{r}^{n+1}-\mathbf{s}^{n+1}\right\|_{\infty} \leq \frac{8\left|\eta^{\prime}\left(2 r_{L}\right)\right|}{\eta^{\prime}\left(r_{U}\right)-5\left|\eta^{\prime}\left(2 r_{L}\right)\right|}|| \mathbf{r}^{n}-\mathbf{s}^{n} \|_{\infty},
$$

where we have from (5.3) that

$$
\frac{8\left|\eta^{\prime}\left(2 r_{L}\right)\right|}{\eta^{\prime}\left(r_{U}\right)-5\left|\eta^{\prime}\left(2 r_{L}\right)\right|}<1
$$

Proof. The first part of the proof will be very similar to the proofs of Lemma 4.4 and Theorem 4.1 , as we will again satisfy the hypotheses of Lemma 4.3. First, we note that (5.3) implies that $r_{U}<a_{1}$ by Lemma 4.4. We prove strict diagonal dominance for $D_{j} \hat{\psi}_{i}^{E}(\mathbf{r})$ given by (5.1). For this argument, we only need to show that $\eta^{\prime}(r)>5\left|\eta^{\prime}(2 s)\right|$ whenever $r, s \in\left(r_{L}, r_{U}\right)$, or since $\eta^{\prime}(2 s)<0$, we need only show

$$
\eta^{\prime}(r)+5 \eta^{\prime}(2 s)>0 \quad \text { for } r, s \in\left(r_{L}, r_{U}\right)
$$

We will need the factor 13 in the hypothesis (5.3) to prove that the mapping is a contraction, which is why the hypothesis is as strong as it is. We further note that from (4.13), (4.14), and the hypothesis (5.4) that we have

$$
\begin{aligned}
\eta^{\prime}(r)+5 \eta^{\prime}(2 s) & >\eta^{\prime}(r)+13 \eta^{\prime}(2 s) \\
& >\eta^{\prime}\left(r_{U}\right)+13 \eta^{\prime}\left(2 r_{L}\right)>0, \quad r, s \in\left(r_{L}, r_{U}\right) .
\end{aligned}
$$

Thus, we have established that $\operatorname{det} D \hat{\mathbf{\Psi}}^{E}(\mathbf{r})>0$ in $\Omega$.

We now verify that $\mathbf{h}(\mathbf{r}, t)$ satisfies the condition $(4.10)$, that is, it does not vanish on $\partial \Omega \times[0,1]$. In the proof of Theorem 4.1, we analyzed $\mathbf{h}(\mathbf{r}, t)$ on $\partial \Omega \times[0,1]$ by grouping the second-neighbor terms. From this point of view (4.17), the iteration problem $\hat{\boldsymbol{\Psi}}^{E}\left(\mathbf{r}^{n+1}\right)+\hat{\mathbf{\Psi}}^{G}\left(\mathbf{r}^{n}\right)=\boldsymbol{\Phi}$, is identical to the problem, $\hat{\boldsymbol{\Psi}}(\mathbf{r})=\boldsymbol{\Phi}$. Thus, we have that

$$
\begin{aligned}
& \min _{\substack{\mathbf{r}^{n+1} \in \partial \Omega \\
r_{j}=r_{U}}} h_{j}\left(\mathbf{r}^{n+1}\right) \geq \min _{\substack{\mathbf{r}^{n+1}, \mathbf{r}^{n} \in \partial \Omega \\
r_{j}=r_{U}}}(1-t)\left[\hat{\psi}_{j}^{L}\left(\mathbf{r}^{n+1}\right)-\Phi_{j}\right]+t\left[\hat{\psi}_{j}^{E}\left(\mathbf{r}^{n+1}\right)+\hat{\psi}^{G}\left(\mathbf{r}^{n}\right)-\Phi_{j}\right] \\
& \geq(1-t)\left[2 \eta\left(2 r_{L}\right)-2 \eta\left(2 r_{U}\right)\right]+\left[\eta\left(r_{U}\right)+4 \eta\left(2 r_{U}\right)-2 \eta\left(2 r_{L}\right)-\Phi_{j}\right]>0, \\
& \max _{\substack{n+1 \in \mathbf{r}_{j} \\
r_{j}=r_{L}}} h_{j}\left(\mathbf{r}^{n+1}\right) \leq \max _{\substack{\mathbf{r}^{n+1}, \mathbf{r}^{n} \in \partial \Omega, r_{j}=r_{L}}}(1-t)\left[\hat{\psi}_{j}^{L}\left(\mathbf{r}^{n+1}\right)-\Phi_{j}\right]+t\left[\hat{\psi}_{j}^{E}\left(\mathbf{r}^{n+1}\right)+\hat{\psi}^{G}\left(\mathbf{r}^{n}\right)-\Phi_{j}\right] \\
& \leq(1-t)\left[2 \eta\left(2 r_{U}\right)-2 \eta\left(2 r_{L}\right)\right]+\left[\eta\left(r_{L}\right)+4 \eta\left(2 r_{L}\right)-2 \eta\left(2 r_{U}\right)-\Phi_{j}\right]<0 .
\end{aligned}
$$

Therefore, we have proven that $\mathbf{h}(\mathbf{r}, t)$ satisfies the condition $(4.10)$, that is, it does not vanish on $\partial \Omega \times[0,1]$. We have now verified the hypotheses of Lemma 4.3 to conclude the existence of a unique solution $\mathbf{r}^{n+1} \in \Omega$ to the iteration equation $(\mathrm{CE})$.

To prove that the mapping (CE) is a contraction, we suppose $\mathbf{r}^{n}, \mathbf{r}^{n+1}, \mathbf{s}^{n}, \mathbf{s}^{n+1} \in \Omega$ satisfy

$$
\begin{aligned}
& \hat{\mathbf{\Psi}}^{E}\left(\mathbf{r}^{n+1}\right)+\hat{\mathbf{\Psi}}^{G}\left(\mathbf{r}^{n}\right)=\boldsymbol{\Phi}, \\
& \hat{\boldsymbol{\Psi}}^{E}\left(\mathbf{s}^{n+1}\right)+\hat{\boldsymbol{\Psi}}^{G}\left(\mathbf{s}^{n}\right)=\boldsymbol{\Phi} .
\end{aligned}
$$

We then have that

$$
\hat{\mathbf{\Psi}}^{E}\left(\mathbf{r}^{n+1}\right)-\hat{\mathbf{\Psi}}^{E}\left(\mathbf{s}^{n+1}\right)=\hat{\mathbf{\Psi}}^{G}\left(\mathbf{s}^{n}\right)-\hat{\mathbf{\Psi}}^{G}\left(\mathbf{r}^{n}\right) .
$$

By the fundamental theorem of calculus, we have that

$$
\begin{aligned}
\hat{\mathbf{\Psi}}^{E}\left(\mathbf{r}^{n+1}\right)-\hat{\boldsymbol{\Psi}}^{E}\left(\mathbf{s}^{n+1}\right) & =L^{E}\left(\mathbf{r}^{n+1}, \mathbf{s}^{n+1}\right)\left(\mathbf{r}^{n+1}-\mathbf{s}^{n+1}\right), \\
\hat{\mathbf{\Psi}}^{G}\left(\mathbf{r}^{n}\right)-\hat{\boldsymbol{\Psi}}^{G}\left(\mathbf{s}^{n}\right) & =L^{G}\left(\mathbf{r}^{n}, \mathbf{s}^{n}\right)\left(\mathbf{r}^{n}-\mathbf{s}^{n}\right),
\end{aligned}
$$


where

$$
\begin{aligned}
L^{E}\left(\mathbf{r}^{n+1}, \mathbf{s}^{n+1}\right) & =\int_{0}^{1} D \hat{\mathbf{\Psi}}^{E}\left(\mathbf{r}^{n+1}+\theta\left(\mathbf{s}^{n+1}-\mathbf{r}^{n+1}\right)\right) \mathrm{d} \theta \\
L^{G}\left(\mathbf{r}^{n}, \mathbf{s}^{n}\right) & =\int_{0}^{1} D \hat{\mathbf{\Psi}}^{G}\left(\mathbf{r}^{n}+\theta\left(\mathbf{s}^{n}-\mathbf{r}^{n}\right)\right) \mathrm{d} \theta
\end{aligned}
$$

We then have by (5.6) and (5.7) that

$$
L^{E}\left(\mathbf{r}^{n+1}, \mathbf{s}^{n+1}\right)\left(\mathbf{r}^{n+1}-\mathbf{s}^{n+1}\right)=-L^{G}\left(\mathbf{r}^{n}, \mathbf{s}^{n}\right)\left(\mathbf{r}^{n}-\mathbf{s}^{n}\right) .
$$

To show that $L^{E}\left(\mathbf{r}^{n+1}, \mathbf{s}^{n+1}\right)$ is nonsingular and estimate its inverse, we define the diagonal matrix, $L^{D}\left(\mathbf{r}^{n+1}, \mathbf{s}^{n+1}\right)$, that contains the dominant nearest-neighbor terms of $L^{E}\left(\mathbf{r}^{n+1}, \mathbf{s}^{n+1}\right)$ by $\left(L^{D}\left(\mathbf{r}^{n+1}, \mathbf{s}^{n+1}\right)\right)_{i j}=$ $L_{i j}^{D}\left(\mathbf{r}^{n+1}, \mathbf{s}^{n+1}\right)$ where

$$
L_{i j}^{D}\left(\mathbf{r}^{n+1}, \mathbf{s}^{n+1}\right)=\left[\int_{0}^{1} \eta^{\prime}\left(r_{i}^{n+1}+\theta\left(s_{i}^{n+1}-r_{i}^{n+1}\right)\right) \mathrm{d} \theta\right] \delta_{i j} .
$$

Since $\mathbf{r}+\theta(\mathbf{s}-\mathbf{r}) \in \Omega=\left(r_{L}, r_{U}\right)^{2 N+1}$ if $\mathbf{r}, \mathbf{s} \in \Omega$ and $\theta \in(0,1)$, we can conclude from (4.13) and (4.14) that

$$
\begin{aligned}
\left\|L^{D}\left(\mathbf{r}^{n+1}, \mathbf{s}^{n+1}\right)^{-1}\right\|_{\infty} & \leq \frac{1}{\eta^{\prime}\left(r_{U}\right)}, \\
\left\|L^{E}\left(\mathbf{r}^{n+1}, \mathbf{s}^{n+1}\right)-L^{D}\left(\mathbf{r}^{n+1}, \mathbf{s}^{n+1}\right)\right\|_{\infty} & \leq 5\left|\eta^{\prime}\left(2 r_{L}\right)\right|,
\end{aligned}
$$

where the matrix norm that is induced by the $\|\mathbf{r}\|_{\infty}$ vector norm is

$$
\|L\|_{\infty}=\max _{i=-N, \ldots, N} \sum_{j=-N}^{N}\left|L_{i j}\right| .
$$

We have that

$$
L^{E}\left(\mathbf{r}^{n+1}, \mathbf{s}^{n+1}\right)=L^{D}\left(\mathbf{r}^{n+1}, \mathbf{s}^{n+1}\right)\left[I+L^{D}\left(\mathbf{r}^{n+1}, \mathbf{s}^{n+1}\right)^{-1}\left(L^{E}\left(\mathbf{r}^{n+1}, \mathbf{s}^{n+1}\right)-L^{D}\left(\mathbf{r}^{n+1}, \mathbf{s}^{n+1}\right)\right)\right],
$$

so it follows from (5.8) that $L^{E}\left(\mathbf{r}^{n+1}, \mathbf{s}^{n+1}\right)$ is nonsingular and we have the estimate

$$
\left\|L^{E}\left(\mathbf{r}^{n+1}, \mathbf{s}^{n+1}\right)^{-1}\right\|_{\infty} \leq \frac{\left\|L^{D}\left(\mathbf{r}^{n+1}, \mathbf{s}^{n+1}\right)^{-1}\right\|_{\infty}}{1-\left\|L^{D}\left(\mathbf{r}^{n+1}, \mathbf{s}^{n+1}\right)^{-1}\right\|_{\infty}\left\|L^{E}\left(\mathbf{r}^{n+1}, \mathbf{s}^{n+1}\right)-L^{D}\left(\mathbf{r}^{n+1}, \mathbf{s}^{n+1}\right)\right\|_{\infty}} .
$$

Hence, can state that

$$
\mathbf{r}^{n+1}-\mathbf{s}^{n+1}=-\left[L^{E}\left(\mathbf{r}^{n+1}, \mathbf{s}^{n+1}\right)\right]^{-1} L^{G}\left(\mathbf{r}^{n}, \mathbf{s}^{n}\right)\left(\mathbf{r}^{n}-\mathbf{s}^{n}\right) .
$$

From (5.2), we can obtain from (4.14) the estimate

$$
\left\|L^{G}\left(\mathbf{r}^{n}, \mathbf{s}^{n}\right)\right\|_{\infty} \leq 8\left|\eta^{\prime}\left(2 r_{L}\right)\right|
$$

so we have from (5.10) and (5.9) that

$$
\begin{aligned}
\left\|\mathbf{r}^{n+1}-\mathbf{s}^{n+1}\right\|_{\infty} & \leq\left\|L^{E}\left(\mathbf{r}^{n+1}, \mathbf{s}^{n+1}\right)^{-1} L^{G}\left(\mathbf{r}^{n}, \mathbf{s}^{n}\right)\right\|_{\infty}\left\|\mathbf{r}^{n}-\mathbf{s}^{n}\right\|_{\infty} \\
& \leq\left\|L^{E}\left(\mathbf{r}^{n+1}, \mathbf{s}^{n+1}\right)^{-1}\right\|_{\infty}\left\|L^{G}\left(\mathbf{r}^{n}, \mathbf{s}^{n}\right)\right\|_{\infty}\left\|\mathbf{r}^{n}-\mathbf{s}^{n}\right\|_{\infty} \\
& \leq \frac{8\left|\eta^{\prime}\left(2 r_{L}\right)\right|}{\eta^{\prime}\left(r_{U}\right)-5\left|\eta^{\prime}\left(2 r_{L}\right)\right|}\left\|\mathbf{r}^{n}-\mathbf{s}^{n}\right\|_{\infty}
\end{aligned}
$$


We have from (5.3) that

so the mapping $\mathbf{r}^{n} \rightarrow \mathbf{r}^{n+1}$ is a contraction and

$$
\frac{8\left|\eta^{\prime}\left(2 r_{L}\right)\right|}{\eta^{\prime}\left(r_{U}\right)-5\left|\eta^{\prime}\left(2 r_{L}\right)\right|}<1
$$

$$
\left\|\mathbf{r}^{n+1}-\mathbf{s}^{n+1}\right\|_{\infty} \leq \frac{8\left|\eta^{\prime}\left(2 r_{L}\right)\right|}{\eta^{\prime}\left(r_{U}\right)-5\left|\eta^{\prime}\left(2 r_{L}\right)\right|}\left\|\mathbf{r}^{n}-\mathbf{s}^{n}\right\|_{\infty} .
$$

We can prove the following corollary of Theorem 5.1 for the ghost force iteration by an argument similar to that used to derive Corollary 4.1 from Theorem 4.1.

Corollary 5.1. Suppose that the external forces, $f_{j}$, are anti-symmetric (3.16), that $r_{L}$ and $r_{U}$ satisfy

$$
\begin{aligned}
& \frac{\hat{r}_{2}}{2}<r_{L}<r_{U}, \\
& \eta^{\prime}\left(r_{U}\right)+13 \eta^{\prime}\left(2 r_{L}\right)>0,
\end{aligned}
$$

and that $\boldsymbol{\Phi}$ satisfies

$$
\eta\left(r_{L}\right)+4 \eta\left(2 r_{L}\right)-2 \eta\left(2 r_{U}\right)<\Phi_{j}<\eta\left(r_{U}\right)+4 \eta\left(2 r_{U}\right)-2 \eta\left(2 r_{L}\right), \quad j=-N, \ldots, N .
$$

Then for every symmetric $\mathbf{r}^{n} \in \Omega=\left(r_{L}, r_{U}\right)^{2 N+1}$ there is a unique symmetric $\mathbf{r}^{n+1} \in \Omega$ such that

$$
F_{j}^{Q C E}\left(\mathbf{r}^{n+1}\right)+F_{j}^{G}\left(\mathbf{r}^{n}\right)+f_{j}=0, \quad j=-N, \ldots, N+1 .
$$

We also have that the induced mapping $\mathbf{r}^{n} \rightarrow \mathbf{r}^{n+1}$ is a contraction and satisfies the inequality

$$
\left\|\mathbf{r}^{n+1}-\mathbf{s}^{n+1}\right\|_{\infty} \leq \frac{8\left|\eta^{\prime}\left(2 r_{L}\right)\right|}{\eta^{\prime}\left(r_{U}\right)-5\left|\eta^{\prime}\left(2 r_{L}\right)\right|}\left\|\mathbf{r}^{n}-\mathbf{s}^{n}\right\|_{\infty},
$$

where we have from (5.11) that

$$
\frac{8\left|\eta^{\prime}\left(2 r_{L}\right)\right|}{\eta^{\prime}\left(r_{U}\right)-5\left|\eta^{\prime}\left(2 r_{L}\right)\right|}<1
$$

The mapping $\mathbf{r}^{n} \rightarrow \mathbf{r}^{n+1}$ converges to the unique symmetric $\mathbf{r}$ in $\Omega$ that satisfies the force-based quasicontinuum equations

$$
F_{j}^{Q C F}(\mathbf{r})+f_{j}=0, \quad j=-N, \ldots, N+1 .
$$

We now apply Corollary 5.1 to the Lennard-Jones potential as in the previous section. This time, we not only need to satisfy the basic inequality (5.3) on $r_{L}$ and $r_{U}$, but we also need to verify that the contraction constant in (5.12) is less than 1 . So, we pick $\gamma \in(0,1)$ and solve for

$$
\frac{8\left|\eta^{\prime}\left(2 r_{L}\right)\right|}{\eta^{\prime}\left(r_{U}\right)-5\left|\eta^{\prime}\left(2 r_{L}\right)\right|}<\gamma
$$

Using the same argument as in Corollary 4.2 , for any $r_{U} \in\left(\hat{r}_{2} / 2, a_{1}\right)$, we choose

$$
r_{L}=\max \left(\frac{\tilde{r}_{2}}{2},\left(\frac{84(5+8 / \gamma)}{256 \eta^{\prime}\left(r_{U}\right)}\right)^{\frac{1}{8}}\right) .
$$

If the resulting $r_{L}$ is less than $r_{U}$, we then have a region for symmetric $\boldsymbol{\Phi}$ for which the iteration is well-defined and a contraction. 
Using the above with contraction constant $\gamma=\frac{1}{2}$, we find that for any symmetric $\boldsymbol{\Phi} \in(-2.56,2.56)^{2 N+1}$ and symmetric $\mathbf{r}^{n} \in(0.9706,1.0771)^{2 N+1}$ there is a unique symmetric $\mathbf{r}^{n+1} \in(0.9706,1.0771)^{2 N+1}$ that satisfies the ghost force iteration equations

$$
F_{j}^{Q C E}\left(\mathbf{r}^{n+1}\right)+F_{j}^{G}\left(\mathbf{r}^{n}\right)+f_{j}=0, \quad j=-N, \ldots, N+1 .
$$

We can finally obtain by taking $\mathbf{s}^{n}=\mathbf{r}$, where $\mathbf{r}$ is the unique symmetric solution to the force-based quasicontinuum equations,

that

$$
F_{j}^{Q C F}(\mathbf{r})+f_{j}=0, \quad j=-N, \ldots, N+1,
$$

$$
\left\|\mathbf{r}^{n+1}-\mathbf{r}\right\|_{\infty} \leq \frac{1}{2}\left\|\mathbf{r}^{n}-\mathbf{r}\right\|_{\infty}
$$

\section{REFERENCES}

[1] S. Antman, Nonlinear problems of elasticity, Applied Mathematical Sciences 107. Springer, New York, second edition (2005).

[2] X. Blanc, C. Le Bris and F. Legoll, Analysis of a prototypical multiscale method coupling atomistic and continuum mechanics. ESAIM: M2AN 39 (2005) 797-826.

[3] X. Blanc, C. Le Bris and P.-L. Lions, Atomistic to continuum limits for computational materials science. ESAIM: M2AN 41 (2007) 391-426.

[4] R.F. Brown, A Topological Introduction to Nonlinear Analysis. Birkhäuser (2004).

[5] W. E and P. Ming, Analysis of multiscale methods. J. Comput. Math. 22 (2004) 210-219.

[6] W. E and P. Ming, Analysis of the local quasicontinuum method, in Frontiers and Prospects of Contemporary Applied Mathematics, T. Li and P. Zhang Eds., Higher Education Press, World Scientific, Singapore (2005) 18-32.

[7] W. E and P. Ming, Cauchy-born rule and the stabilitiy of crystalline solids: Static problems. Arch. Ration. Mech. Anal. 183 (2007) 241-297.

[8] W. E, J. Lu and J. Yang, Uniform accuracy of the quasicontinuum method. Phys. Rev. B 74 (2006) 214115.

[9] W. Fleming, Functions of Several Variables. Springer-Verlag (1977).

[10] J. Knap and M. Ortiz, An analysis of the quasicontinuum method. J. Mech. Phys. Solids 49 (2001) 1899-1923.

[11] P. Lin, Theoretical and numerical analysis for the quasi-continuum approximation of a material particle model. Math. Comp. 72 (2003) 657-675 (electronic).

[12] P. Lin, Convergence analysis of a quasi-continuum approximation for a two-dimensional material. SIAM J. Numer. Anal. 45 (2007) 313-332.

[13] M. Marder, Condensed Matter Physics. John Wiley \& Sons (2000).

[14] R. Miller and E. Tadmor, The quasicontinuum method: Overview, applications and current directions. J. Comput. Aided Mater. Des. 9 (2002) 203-239.

[15] R. Miller, L. Shilkrot and W. Curtin, A coupled atomistic and discrete dislocation plasticity simulation of nano-indentation into single crystal thin films. Acta Mater. 52 (2003) 271-284.

[16] J.T. Oden, S. Prudhomme, A. Romkes and P. Bauman, Multi-scale modeling of physical phenomena: Adaptive control of models. SIAM J. Sci. Comput. 28 (2006) 2359-2389.

[17] C. Ortner and E. Süli, A posteriori analysis and adaptive algorithms for the quasicontinuum method in one dimension. Technical report, Oxford Numerical Analysis Group (2006).

[18] C. Ortner and E. Süli, A priori analysis of the quasicontinuum method in one dimension. Technical report, Oxford Numerical Analysis Group (2006).

[19] S. Prudhomme, P.T. Bauman and J.T. Oden, Error control for molecular statics problems. Int. J. Multiscale Comput. Eng. 4 (2006) 647-662.

[20] D. Rodney and R. Phillips, Structure and strength of dislocation junctions: An atomic level analysis. Phys. Rev. Lett. 82 (1999) 1704-1707.

[21] D. Serre, Matrices: Theory and applications, Graduate Texts in Mathematics 216. Springer-Verlag, New York (2002). Translated from the 2001 French original.

[22] V. Shenoy, R. Miller, E. Tadmor, D. Rodney, R. Phillips and M. Ortiz, An adaptive finite element approach to atomic-scale mechanics - the quasicontinuum method. J. Mech. Phys. Solids 47 (1999) 611-642.

[23] T. Shimokawa, J. Mortensen, J. Schiotz and K. Jacobsen, Matching conditions in the quasicontinuum method: Removal of the error introduced at the interface between the coarse-grained and fully atomistic regions. Phys. Rev. B 69 (2004) 214104.

[24] E. Tadmor, M. Ortiz and R. Phillips, Quasicontinuum analysis of defects in solids. Phil. Mag. A 73 (1996) $1529-1563$. 